\title{
Obsolescencia de la envolvente térmica y acústica de la vivienda social de la postguerra española en áreas urbanas vulnerables. El caso de Zaragoza
}

\author{
Energy and acoustics related obsolescence of social housing of Spain's post- \\ war in less favoured urban areas. The case of Zaragoza \\ F. $\operatorname{Kurtz}^{(*)}$, M. Monzón ${ }^{(*)}, \underline{\text { B. López-Mesa }}{ }^{(*)}$
}

\section{RESUMEN}

En las ciudades españolas actuales, la vivienda de protección pública construida durante la etapa posterior a la Guerra Civil y hasta mediados de los 60 constituye un modelo ampliamente extendido. En este artículo se realiza un diagnóstico de la obsolescencia física de la envolvente térmica y acústica de 21 Conjuntos Urbanos de Interés de Zaragoza vulnerables de la posguerra española, definiendo de forma sistemática las soluciones constructivas empleadas y comparando su comportamiento energético y acústico con los estándares actuales. Se observa que el sistema envolvente semitransparente es débil tanto acústica como térmicamente, y que el sistema envolvente opaco presenta fuertes deficiencias a nivel energético particularmente en el caso de cubiertas.

Palabras clave: Vivienda social; rehabilitación arquitectónica; pobreza energética; transmitancia térmica; aislamiento frente al ruido aéreo.

\section{ABSTRACT}

In current Spanish cities, social housing built after the Civil War represents a widely spread building typology. In this paper we diagnose the physical obsolescence of the thermal and acoustic envelope of 21 residential units in less favoured urban areas of Zaragoza built in Spain's post-war. The existing constructive solutions are defined, and their energy and acoustic behaviours are compared to current standards. It is observed that widows show poor performance regarding energy and acoustics, and that the opaque part of the envelope of these buildings is insufficient regarding energy standards, especially the roofs.

Keywords: Social housing; building refurbishment; energy poverty; U-value; airborne sound reduction.

(*) Universidad de Zaragoza. Zaragoza (España).

Persona de contacto/Corresponding author: belinda@unizar.es (B. López-Mesa)

Cómo citar este artículo/Citation: Kurtz, F., Monzón, M., López-Mesa, B. (2015). Obsolescencia energética y acústica de la vivienda social de la postguerra española en áreas urbanas vulnerables. El caso de Zaragoza. Informes de la Construcción, 67(EXTRA-1): mo21, doi: http://dx.doi.org/10.3989/ic.14.062.

Licencia/License: Salvo indicación contraria, todos los contenidos de la edición electrónica de Informes de la Construcción se distribuyen bajo una licencia de uso y distribución Creative Commons Reconocimiento no Comercial 3.o. España (cc-by-nc). 


\section{INTRODUCCIÓN}

Tras la Guerra Civil, numerosas ciudades españolas debían ser reconstruidas, y al mismo tiempo, debían prepararse para recibir un constante y creciente flujo migratorio del campo, donde la situación era aún más precaria. Así, la ley del 19 de abril de 1939, por la que se creó el Instituto Nacional de la Vivienda y el Régimen de Protección de La Vivienda, se aprobó para la construcción de «viviendas higiénicas», con el objetivo de responder al problema del alojamiento de una creciente población empobrecida en las ciudades y bajo el influjo del discurso higienista iniciado a mediados del siglo xIx. Actualmente la vivienda de protección pública construida durante la etapa posterior a la Guerra Civil y hasta mediados de los 60 constituye un modelo ampliamente extendido que se ideó en uno de los periodos de más profunda depresión económica en España. Desde la construcción de estos conjuntos de viviendas, no se han realizado mejoras sustanciosas en los edificios que las albergan ni en sus entornos urbanos inmediatos, salvo casos aislados.

En los 90, la Organización para la Cooperación y el Desarrollo Económicos (OCDE) comenzó a llamar la atención sobre los barrios desfavorecidos de las ciudades alegando que dificultan el crecimiento económico, debilitan la cohesión social y suponen altos costes medioambientales (1). Se publicaron varios informes, como el de Integración de barrios desfavorecidos (1) en el que se analizaban las políticas en curso en distintos países de la OCDE y se recomendaron líneas de actuación multisectoriales en función de los problemas específicos. De esta forma, en la Agenda 2000 de la Unión Europea (2), los barrios desfavorecidos cobraron importante atención, y en los Estados Miembros se desarrollaron estudios específicos, como el del Ministerio de Fomento dirigido por Arias Goytre en 2000 (3), en el que se detectaron en los municipios de más de 50.000 habitantes 374 barrios desfavorecidos, entre los cuales los conjuntos de Promociones de Vivienda Unitarias eran los que más habitantes albergaban y se caracterizaban por tener la peor situación socio-laboral. En el mencionado estudio se instaba a seguir identificando barrios desfavorecidos.

En 2004, el Ayuntamiento de Zaragoza a través de la Sociedad Municipal Zaragoza Vivienda (SMZV, entonces Sociedad Municipal de Rehabilitación Urbana de Zaragoza) inició el estudio y propuestas de rehabilitación de 22 conjuntos de viviendas sociales con el objetivo de disponer de «una radiografía social y física» (4) de todo ese patrimonio edificado, con Rubio del Val como coordinador y con la implicación mediante concurso de equipos pluridisciplinares de profesionales compuestos por arquitectos, sociólogos, trabajadores sociales, abogados, economistas, etc. Estos 22 conjuntos habían sido proyectados en el periodo 1942-1964 y construidos en el periodo 1945-1975, promovidos por diversos organismos públicos (Obra Sindical del Hogar, Patronato Municipal de la Vivienda), por organismos privados sin ánimo de lucro (Patronatos religiosos) o por algunas empresas para sus trabajadores (Tranvías de Zaragoza, etc.), y habían quedado declarados Conjuntos Urbanos de Interés (CUI) en el Plan General de Ordenación Urbana de Zaragoza (PGOUZ) de 2001 -a excepción de dos de ellos-, por su carácter de unidad reuniendo valores ambientales representativos de tipos de edificación residencial y de agrupación urbana característicos de su época (5), como pueden ser la racionalidad tipológica de las soluciones, los espacios libres definidos por los bloques y el empleo de materiales tradicionales (4). En 2006, la SMZV puso en marcha los trabajos para el desarrollo de las propuestas de rehabilitación, declarando algunos conjuntos Áreas de Rehabilitación Integral (ARI) al amparo de los Planes de Vivienda estatal y autonómicos entonces vigentes. Dentro de estos conjuntos varios bloques en el período 2008-2011 han sido objeto de una rehabilitación integral con motivos principalmente energéticos y de accesibilidad, la cual únicamente afecta a la envolvente del edificio y a las zonas comunes. Posteriormente se han redactado otros proyectos de rehabilitación integral, alguno ya iniciado. Todas estas rehabilitaciones se señalan en la Figura 1.

Por otro lado, la vertiente medioambiental del discurso sostenibilista iniciado en los años 60 del siglo $\mathrm{xx}$, ha ido cobrando fuerza a través de los compromisos internacionales de reducción de gases de efecto invernadero (GEI) y ha ido incorporando un desarrollo específico para los diferentes sectores de actividad, como el edificatorio, que recientemente ha reconocido la relevancia de centrar la mirada en la renovación de la ciudad construida. La disminución del impacto ambiental del sector de la edificación radica en la renovación del parque existente, así como en impulsar un nuevo ciclo urbano basado en el equilibrio entre los factores sociales, ambientales y económicos. Así, según la reciente directiva 2012/27/UE, los Estados miembros deben renovar cada tres años una estrategia a largo plazo para movilizar inversiones en la renovación del parque nacional, para la que es importante disponer información al servicio de las Administraciones públicas relativa a la diagnosis del parque edificatorio, a la rentabilidad de las actuaciones de renovación, a protocolos de renovación profunda por fases y a sus medidas de los beneficios energéticos y de mayor amplitud. Asimismo en España, en abril de 2013, se aprobó el proyecto de Ley de rehabilitación, regeneración y renovación urbanas ( $\left.\mathrm{L}_{3} \mathrm{R}\right)$, donde se hace alusión a la creación y actualización permanente de «información al servicio de las políticas públicas para un medio urbano sostenible» relativa a la diagnosis del parque edificatorio y a mapas de barrios vulnerables como una parte importante de los futuros instrumentos al servicio de las nuevas políticas de rehabilitación urbana. La diagnosis del parque edificatorio se convierte así en un elemento esencial reconocido para el nuevo ciclo urbano que dirige su mirada en la renovación de la ciudad construida.

En los últimos años varios estudios han abordado esta temática, basándose fundamentalmente en la obtención de los consumos energéticos y emisiones de $\mathrm{CO} 2$ del parque edificatorio existente y sus posibilidades de mejora. Todos ellos seleccionan muestras edificatorias, de escala europea, nacional o regional, representativas del parque edificatorio que estudian y las extrapolan al resto de edificios. Existen, no obstante, dos grandes aproximaciones: a) Los que realizan el estudio de consumos energéticos a partir de las estadísticas de consumo de edificación residencial y posteriormente reparten dichos consumos totales entre las viviendas, fundamentalmente a través de la simulación a partir de parámetros sobre la morfología edificatoria, las características constructivas -en función del año de construcción- y la zona climática (por ejemplo: (6) (7) (8) (9) (10)); y b) Los que detectan los edificios más característicos a partir de las estadísticas de consumo de edificación residencial para posteriormente estudiar los consumos de estos edificios a través de entrevistas (por ejemplo: 11). En ambas aproximaciones, se obtiene una perspectiva generalizada de los consumos del parque edifi- 
catorio de interés para cálculos globales, pero menos indicada para hacer zoom en tipologías concretas debido a que su cálculo está basado en la repartición proporcional de los consumos energéticos oficiales por viviendas en las secciones censales de cada tipología considerada, dejando la dispersión generada por otros factores -como el efecto de los ocupantes del edificio- quede absorbida por su distribución uniforme sobre el parque. Los efectos de los ocupantes más reconocidos en la literatura son el uso más o menos eficiente que los usuarios hacen de las instalaciones y la pobreza energética. El uso afecta aproximadamente al 4,2 \% del consumo energético frente al $42 \%$ que suponen las características del edificio (12), por lo que parece aceptable obviarlo, sin embargo en el presente estudio no podemos obviar la pobreza energética, ya que esta afecta al $19 \%$ de los hogares aragoneses (13), y presumiblemente representará un porcentaje mayor en la vivienda social de barrios vulnerables. En este sentido, LópezMesa et al. (14) encuentran que en las viviendas rehabilitadas de las que han podido recopilar facturas en el Grupo Girón en Zaragoza los ahorros energéticos reales son prácticamente nulos, lejos del $52 \%$ que se augura a partir de los datos arrojados por los programas de simulación. Con la rehabilitación de dichas viviendas los usuarios no ahorran, aunque sí ganan en confort como confirman algunos entrevistados (14). En la misma línea Sendra et al. (15) encuentran que en edificios de viviendas protegidas en el Sur de España no existe habitualmente una relación directa entre demanda de energía y uso real de la misma, asociado el bajo consumo a una carencia de prestaciones. Podemos achacar este hecho a varias cuestiones, entre ellas, la situación de pobreza energética de los usuarios de viviendas sociales, que les conduce a consumir energía en función de su disponibilidad presupuestaria.

Este artículo se centra en la diagnosis de la envolvente térmica y acústica de los CUI de vivienda social de Zaragoza vulnerables, entendiendo por conjunto vulnerable aquél que «se encuentra frente a una posible situación crítica, de forma que, de no actuarse sobre las bases del conflicto, el área entrará en crisis, pudiéndose producir una degradación funcional y social del ámbito que lo conduzca a la marginación» (16). En el estudio dirigido por Rubio del Val (4) ya se realizó un diagnóstico del medio físico y de la población de estos barrios vulnerables. El diagnóstico del medio físico incluyó: una clasificación sistemática de la edificación en función de los tipos de bloques, portales y viviendas; la definición geométrica en planta de los mismos y fichas por bloque de las principales superficies y dimensiones de la urbanización, bloques, portales, viviendas, fachadas y escaleras; el estudio de la inadecuación de la edificación respecto a la normativa entonces vigente a través de fichas que describen en forma de texto las soluciones constructivas y los incumplimientos; la tipificación de los problemas y diagnóstico de la edificación que consistió en un listado exhaustivo por sistemas de edificación y sus elementos; y la tipificación de las soluciones y propuestas de actuación a través de una base de datos que incluía una base de precios.

En este artículo el objetivo es realizar un diagnóstico sistemático de la obsolescencia física -acústica e higrotérmica- de la envolvente de los CUI de Zaragoza medida como la diferencia entre el comportamiento actual de las envolventes y la situación considerada de confort en la normativa, ya que este factor es el que de forma más fiable se puede medir en el total de los conjuntos de viviendas fuertemente afectadas por la pobreza energética. Simulaciones de los consumos no arrojan datos fiables como hemos comentado anteriormente.
El enfoque propuesto en este artículo consistente en estudiar el comportamiento de los elementos de las envolventes por separado permite asimismo la identificación de los elementos prioritarios en las rehabilitaciones. Se han tomado 21 conjuntos -de los 22 originalmente estudiados (4) - usando como criterio de selección que sean Conjuntos Urbanos de Interés (zona C) en el PGOUZ de 2007 (17), que hayan sido proyectados en el período 1942-1964 y que sean considerados barrios vulnerables (4). Los objetivos específicos del artículo son la definición de forma gráfica de las soluciones constructivas de la envolvente de los edificios que no han sido objeto de rehabilitaciones integrales de los 21 conjuntos residenciales (dado que la información gráfica existente es poco precisa y dispersa) y su estudio cronológico, el estudio estadístico de los huecos de ventana en la actualidad, la valoración acústica e higrotérmica de los elementos de la envolvente, y su comparación con la normativa vigente en la actualidad. Los resultados de este artículo resultan, por tanto, complementarios a los del estudio de Rubio del Val (4). Asimismo este estudio resulta útil para futuros análisis, de manera que en los estudios de rentabilidad de las actuaciones de renovación, en las propuestas de protocolos de renovación profunda por fases y en las medidas de los beneficios energéticos, se pueda contar con datos con rigor suficiente.

\section{METODOLOGÍA DE INVESTIGACIÓN}

La metodología de trabajo se ha compuesto de los siguientes pasos:

1. Definición gráfica de soluciones de la envolvente.

1.a Recopilación sistemática de la información del estado original y posibles rehabilitaciones de las edificaciones de cada uno de los 21 CUI disponibles en el archivo municipal de Zaragoza, en el archivo del palacio de Montemuzo, PGOUZ de 2007 (17), así como en otras fuentes bibliográficas (4) (18) (19) (20).

1.b Definición gráfica de tipologías de detalles constructivos de la envolvente incluyendo su localización en las edificaciones de los diferentes conjuntos residenciales y análisis de su extensión de uso.

2. Estudio estadístico de los huecos de ventana en la actualidad.

2.a Recogida inicial pasiva de datos de huecos de ventana de dos bloques de viviendas en el conjunto de José Antonio Girón mediante observación de las fachadas desde el exterior y su correspondiente captura fotográfica en el mes de abril de 2014.

2.b Puesta en común de las fotografías y definición de los parámetros a observar en lo sucesivo:

- Composición de ventana: sencilla o doble.

- Tipos de persiana.

- Tipos de cierre: batiente o corredera.

- Materiales de carpintería.

- Tipos de vidrio: sencillo o doble.

- Color de carpintería.

2.c Definición del tamaño de la muestra a observar. La población de huecos de ventana en el total de las 7.900 vivien- 
das de los 21 CUI es de aproximadamente 47.500 unidades. Para garantizar la representatividad de todos los conjuntos, decidimos estudiar al menos un bloque de viviendas de cada conjunto, lo que estimamos que supondría una muestra, incluyendo los dos primeros bloques observados, de unos 2.500-3.00o huecos. Nos encontramos ante un muestreo aleatorio estratificado y conglomerado, en la que cada CUI representa un estrato del que se estudia un conglomerado -el bloque-, es decir, una unidad de elementos que contiene representantes de toda la población. Para saber si los 2.500-3.00o huecos nos dan la fiabilidad suficiente, recurrimos a la ecuación para el cálculo del tamaño de la muestra para poblaciones finitas cuando $\mathrm{n} / \mathrm{N}>0,05$, donde $\mathrm{n}$ es el tamaño de la muestra y $\mathrm{N}$ la población:

$$
n=\frac{N \cdot Z^{2} \cdot p \cdot q}{e^{2} \cdot(N-1)+Z^{2} \cdot p \cdot q}
$$

Donde:

- Z es el nivel deseado de fiabilidad, igual a 3, para un 99,7\%.

- p y q representan la variabilidad de los fenómenos a estudiar, que consideramos desconocida a priori, por lo que damos a ambos valores el valor de $50 \%$.

- e representa el error muestral que estimamos en el $5 \%$.

$\mathrm{Al}$ estar el estudio diseñado como una muestra por conglomerados (una selección representativa de bloques) y no como una muestra aleatoria simple, el tamaño de la muestra se multiplica por el efecto de diseño (D), que en el muestreo por conglomerados se estima en 1,5. De esta forma obtenemos un tamaño de muestra de 1.767 huecos de ventana, por lo que las 2.500-3.00o observaciones inicialmente previstas son suficientes para generalizar a toda la población los resultados.

2.d Recogida de datos de huecos de ventana durante abril y mayo de 2014. Llevada a cabo por dos observadores para todos los conjuntos excepto tres con un único observador, se realizó tomando notas in situ y realizando fotografías. Los huecos de ventana que no estaban accesibles a la vista cuando se realizó el estudio y aquellos que presentaban algunas características que suscitaban dudas han sido codificados convenientemente. El número total de huecos observados ascendió a 2.672.

2.e Clasificación y codificación de los tipos de huecos encontrados, y obtención de los porcentajes de cada solución.

3. Valoración higrotérmica y acústica de los elementos opacos de la envolvente.

3.a Cálculo de la transmitancia térmica de los elementos opacos de la envolvente conforme a la metodología en el Documento de Apoyo DA DB-HE / 1 (21) y su comparación con los valores límite de transmitancia recogidos en la sección HE1 Limitación de la demanda energética del Documento Básico DB-HE Ahorro de energía (DB-HE1) para las rehabilitaciones. Los valores de conductividad térmica de los materiales se han obtenido del Catálogo de Elementos Constructivos del CTE (CEC-CTE) (22).

3.b Comprobación de condensaciones usando el método del factor de temperaturas superficiales de acuerdo con el DA DB-HE / 2 (23). El factor de temperatura de la superfi- cie interior se ha calculado para la ciudad de Zaragoza tomando como temperatura exterior mínima media del mes de enero $6,2^{\circ} \mathrm{C}$, temperatura interior $20^{\circ} \mathrm{C}$ y humedad relativa interior $55 \%$.

3.c Estudio de las exigencias del Documento Básico DBHR Protección frente al ruido (DB-HR) si se aplicaran a los conjuntos residenciales.

3.d Predicción del Índice global de reducción acústica, ponderado A, para ruido exterior dominante de automóviles o de aeronaves $\left(\mathrm{R}_{\mathrm{A}, \mathrm{tr}}\right)$ de los elementos opacos de la envolvente siguiendo la ley de masas con las mejoras de Cremer, Sewer y Sharp para sistemas constructivos sin cámara de aire y las ecuaciones de Sharp, Fahy y Rindel para sistemas con cámaras (24) mediante el uso del software de predicción acústica Insul 7.0.6 (25).

4. Valoración acústica de los elementos de la envolvente.

4.a Consulta de las características higrotérmicas de las ventanas sencillas en el CEC-CTE (22) y cálculo de la transmitancia de las ventanas dobles según la norma UNE-EN ISO 6946:2007 (26).

4.b Valoración del $\mathrm{R}_{\mathrm{A}, \mathrm{tr}}$ de los elementos semitransparentes usando dos aproximaciones. Por un lado, se han recogido los datos del CEC-CTE y por otro hemos predecido el valor, para una ventana media de 0,9x1,2 m, según el método recogido en el estudio de apoyo para el CTE por Moreno y Peña (27), consistente en calcular el $\mathrm{R}_{\mathrm{A}, \mathrm{tr}}$ del hueco según la norma UNE EN 12354 y mejorar su concordancia con la realidad constructiva española mediante la consideración de una superficie equivalente de rendija de transmisividad igual a 1 , cuyo espesor es $1,5 \mathrm{~mm}$ para las holguras entre marco y hoja y entre hojas, y $8 \mathrm{~mm}$ para el capialzado cuando éste es no prefabricado.

\section{RESULTADOS}

Las 7.900 viviendas recogidas en la Figura 1 se distribuyen por barrios y grupos, que en la época en que se construyeron formaron parte de la periferia de la ciudad. La mayoría de los conjuntos están constituidos por bloques aislados de dos crujías y ventilación cruzada. Otros se disponen formando manzanas semiabiertas o cerradas; en forma de peine -como el grupo Puente Virrey Tejar-; generando patios de luces interiores -grupo Santa Rosa-; o en altura -como es el caso de las torres de Aloy Sala y Balsas de Ebro Viejo- (Figura 1). En un número importante de estos conjuntos la orientación predominante es norte-sur, justificándose en los proyectos por motivos de soleamiento y protección frente a los vientos de los espacios libres entre los bloques. La Figura 2 ofrece imágenes de los diferentes conjuntos.

\subsection{Definición gráfica de las soluciones constructivas de la envolvente}

La solución de fachada que más se repite en los 21 conjuntos es la de ladrillo macizo caravista de diferentes espesores que van desde un pie y medio hasta medio pie (Figura 3). En la mayoría de los casos las fachadas trabajan como muros de carga. En muchos de los bloques estudiados con esta solución, las fachadas van reduciéndose de espesor a medida que crecen en altura, rematando la última planta con una solu- 


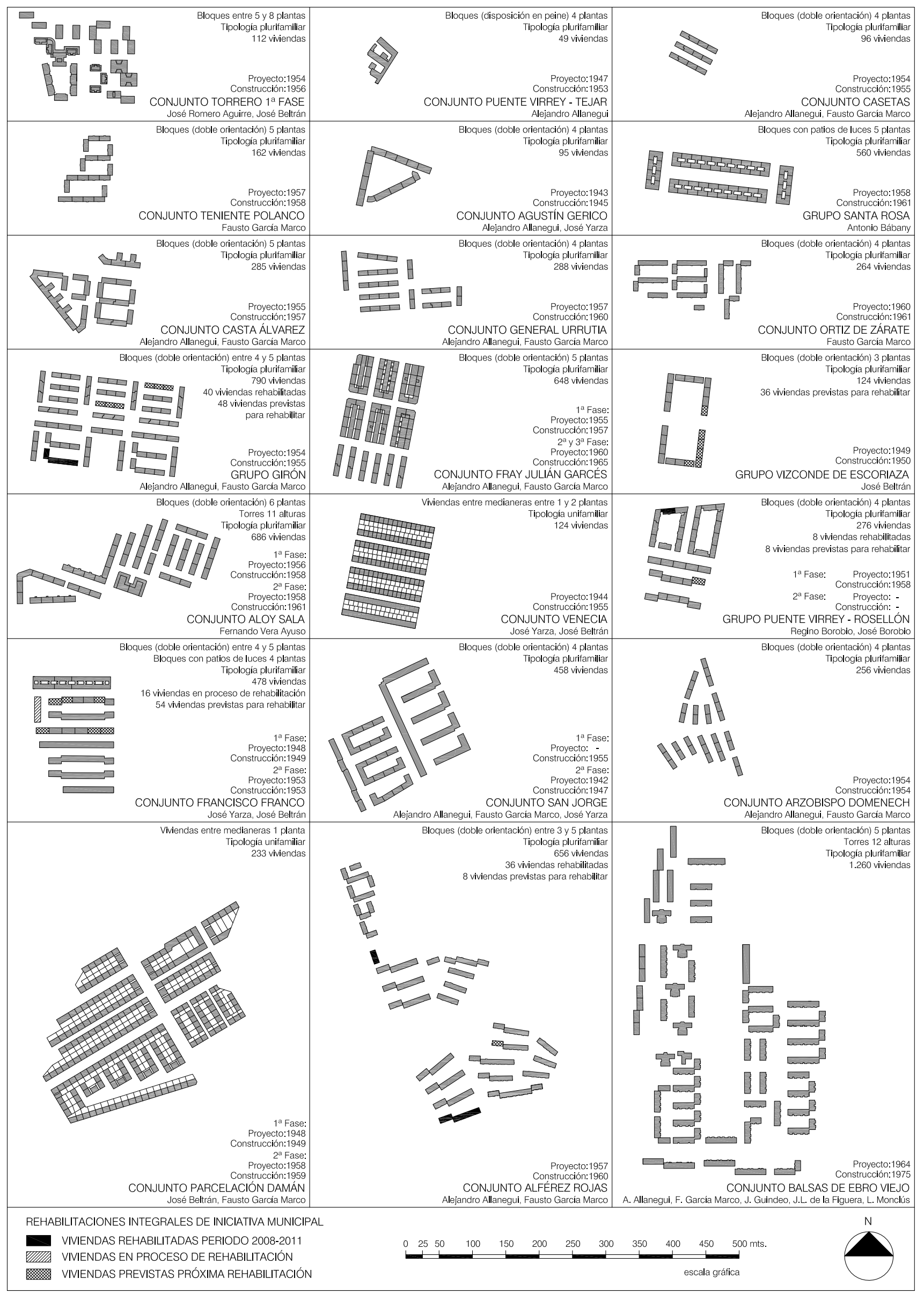

Figura 1. Diagramas y principales características de los 21 conjuntos en planta, señalando los bloques rehabilitados, en periodo de rehabilitación, y previstos para próximas rehabilitaciones en cada conjunto. 

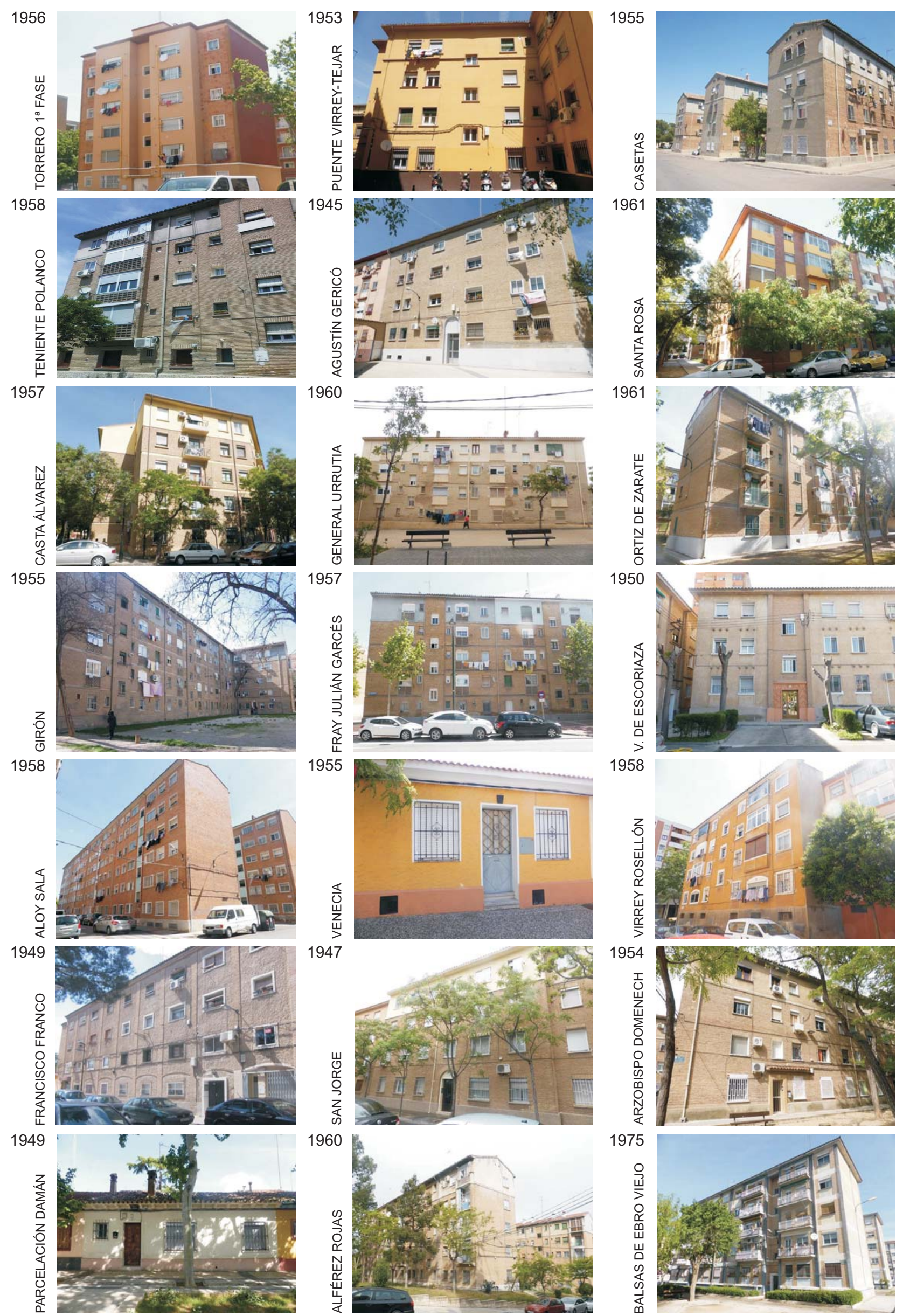

Figura 2. Imágenes de los conjuntos. 

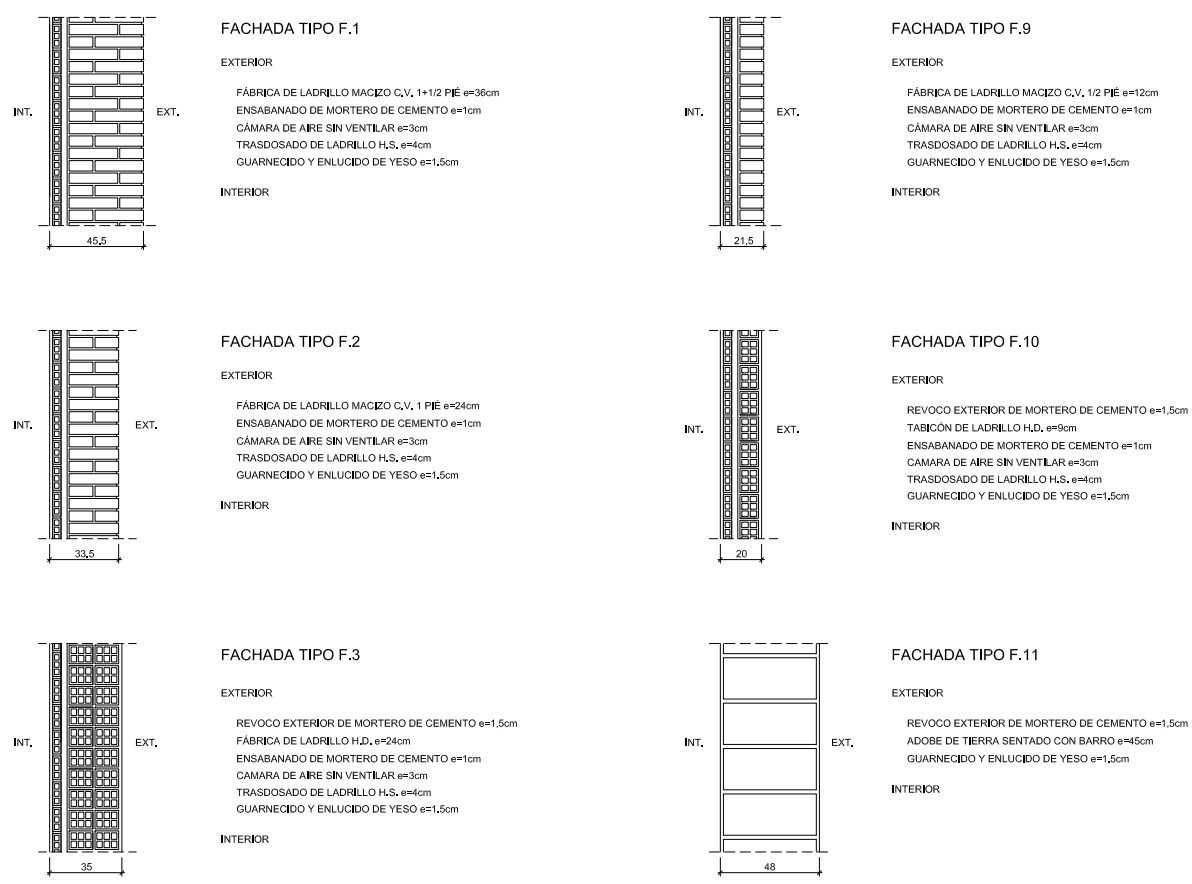

FACHADA TIPO F.11

EXTERIOR

REVOCO EXTERIOR DE MORTERO DE CEMENTO E=1,5cm ADOBE DE TIERRA EENTADO CON BARRO $\mathrm{e}=45 \mathrm{C}$. GUARNECIDO Y ENLUCIDO DE YESO e=1.15CM INTERIOR
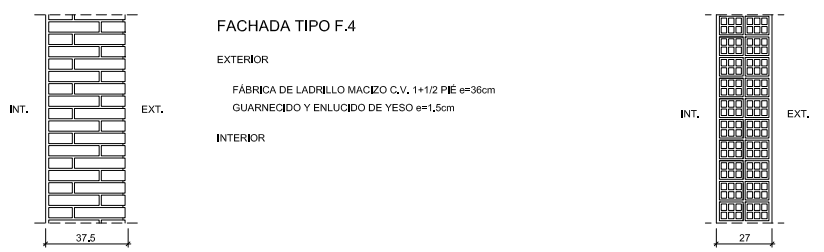

FACHADA TIPO F.12

EXTERIOR REVOCO EXTERIOR DE MORTERO DE CEMENTO e=1,5cn INTERIOR

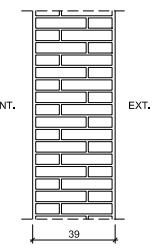

FACHADA TIPO F.5

EXTERIOR

REVOCO EXTERIOR DE MORTERO DE CEMENTO e1,50m

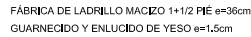

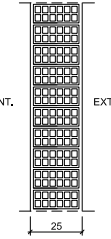

FACHADA TIPO F.13 GuAFI
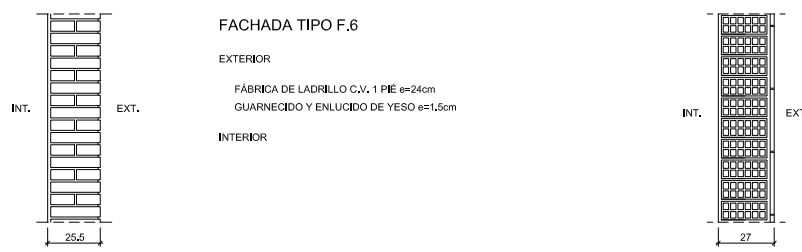

FACHADA TIPO F.14

EXTERIOR

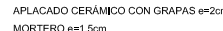
INTERIOR FABRLA DE LADRLLLO HUECO GIGANTE E=220M INTERIOR
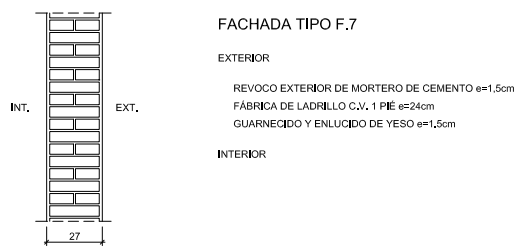

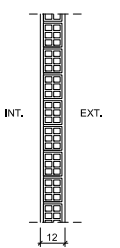

FACHADA TIPO F. 15

EXTERIOR REVOCO EXTERIOR DE MORTERO DE CEMENTO e $=1,5 \mathrm{~cm}$

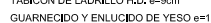

INTERIOR

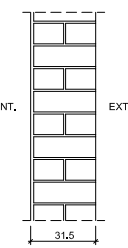

FACHADA TIPO F.8

EXTERIOR

FABRLCA DE BLOQUE MACIZO DE HORMGON $\mathrm{e}=30 \mathrm{CM}$
GUARNECIDO Y ENLUCIDO DE YYSO

INTERIOR

Figura 3. Soluciones constructivas de fachada en los 21 CUI. 
ción de fábrica de ladrillo hueco doble de un pie de espesor jaharrado al exterior y enlucido al interior. Existen casos singulares como la $1^{\mathrm{a}}$ fase del grupo Francisco Franco, donde sus fachadas están realizadas con adobe de tierra sentado con barro. En este caso la estructura se resuelve con pilares de fábrica de ladrillo macizo y muros transversales de carga entre la estructura de soportes. Otro caso es el de las viviendas unifamiliares de la parcelación Damán y los bloques del grupo Agustín Gericó, donde se resuelve la estructura de muros de carga y cerramiento mediante fábrica de bloque de hormigón de $30 \mathrm{~cm}$ de espesor. Otra solución singular lo constituye el conjunto Torrero donde las fachadas son de fábrica de ladrillo hueco «gigante» aplacado con baldosas cerámicas ancladas con grapas. En el conjunto Balsas de Ebro Viejo el cerramiento se ha resuelto con doble hoja de fábrica de ladrillo perforado caravista con cámara de aire cerrada mediante tabique de fábrica de ladrillo hueco sencillo, sustituyéndose la hoja exterior por fábrica de ladrillo hueco doble en las zonas jaharradas con mortero.

La mayoría de los suelos en contacto con el terreno se han realizado sobre solera de hormigón en masa de $10 \mathrm{~cm}$ de espesor, donde en algunos casos, se le ha dado un tratamiento hidrófugo o impermeabilizante (Figura 4). En el conjunto Balsas de Ebro Viejo se ha resuelto mediante forjado sanitario, elevando la planta baja aproximadamente un metro sobre la rasante de la acera. También están provistos de forjado sanitario aquellos bloques del grupo Alférez Rojas donde el relleno de la planta baja es superior a $60 \mathrm{~cm}$. Podemos encontrar otros tipos de suelos en los conjuntos Torrero, Aloy Sala o las torres de Balsas de Ebro Viejo donde las plantas bajas se utilizan como locales comerciales, siendo su cerramiento horizontal el forjado del techo de la planta baja. Esto mismo ocurre en los grupos provistos de planta sótano como son los grupos Agustín Gericó o Aloy Sala.

Las cubiertas en general son inclinadas a dos o cuatro aguas, excepto en el caso de las torres que son planas a la catalana (Figura 5). Las de dos aguas están muy ventiladas, y su cerramiento horizontal se resuelve mediante el forjado o bien de viguetas cerámicas armadas o de hormigón. Las de cuatro aguas no están ventiladas, resolviéndose generalmente los forjados de cubierta con viguetas cerámicas o rollizos de madera, excepto algunos grupos que han utilizado forjados de hormigón armado aligerados. Un caso singular es el de la $1^{\mathrm{a}}$ fase del grupo Francisco Franco donde el techo de la última planta se resuelve como un forjado más de bóveda tabicada y sobre éste se tiende la cubierta inclinada de estructura de rollizos, cañizo y torta de barro, sobre el que se asienta las teja cerámica tipo árabe.

\subsection{Estudio estadístico de los huecos de ventana en la actualidad}

Se han encontrado los tipos de ventanas que se presentan a continuación, a los que se le ha dado un código entre paréntesis que permite su identificación en la Figura 6 y Tabla 1. Cuando algunas características de la ventana no eran accesibles a la vista, se recogieron los datos que sí eran observables, y lo no observable se ha codificado como «d» de desconocido. La siguiente clasificación muestra los tipos:

\section{- Ventana sencilla}

- Sin sombreamiento exterior

- Corredera de aluminio (S.1.C)
- Abatible de aluminio (S.1.A)

- Con sombreamiento exterior

- Persiana enrollable:

- Con cajón interior tipo monoblock (S.2a)

SUELOS TIPO S.1

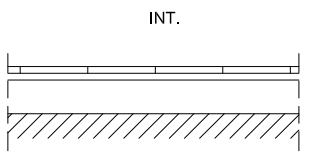

EXT.

INTERIOR

BALDOSA HIDRÁULICA $e=2 \mathrm{~cm}$ CAMISA DE MORTERO $\mathrm{e}=2 \mathrm{~cm}$ SOLERA DE HORMIGÓN $\mathrm{e}=10 \mathrm{~cm}$

EXTERIOR

SUELOS TIPO F.TS.1

INT.

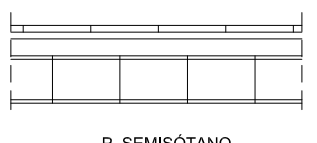

INTERIOR

BALDOSA HIDRÁULICA $e=2 \mathrm{~cm}$ CAMISA DE MORTERO $\mathrm{e}=2 \mathrm{~cm}$ FORJADO DE H.A. VIGUETAS "IN SITU" PLANTA SEMISÓTANO

SUELOS TIPO F.TS.2

INTERIOR

BALDOSA HIDRÁULICA $e=2 \mathrm{~cm}$ CAMISA DE MORTERO $\mathrm{e}=2 \mathrm{~cm}$ FORJADO DE H.A. VIGUETAS PREFABRICADAS

P. SEMISÓTANO

PLANTA SEMISÓTANO

\section{SUELOS TIPO F.S.1}

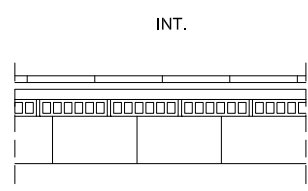

INTERIOR

BALDOSA HIDRÁULICA $e=2 \mathrm{~cm}$

CAMISA DE MORTERO $\mathrm{e}=2 \mathrm{~cm}$

FORJADO DE VIGUETAS CERÁMICAS ARMADAS CAPA DE COMPRESIÓN DE HORMIGÓN $\mathrm{e}=3 \mathrm{~cm}$ TABLERO DE RASILLA $\mathrm{e}=5 \mathrm{~cm}$

CÁMARA DE AIRE VENTILADA

SUELOS TIPO F.TB.1

INT.

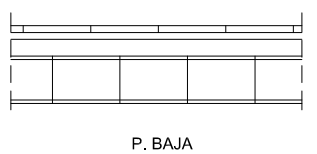

INTERIOR

BALDOSA HIDRÁULICA $\mathrm{e}=2 \mathrm{~cm}$ CAMISA DE MORTERO $\mathrm{e}=2 \mathrm{~cm}$ FORJADO DE VIGUETAS METÁLICAS

PLANTA BAJA

SUELOS TIPO F.TB.2

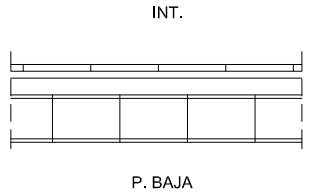

INTERIOR

BALDOSA HIDRÁULICA $e=2 \mathrm{~cm}$ CAMISA DE MORTERO $\mathrm{e}=2 \mathrm{~cm}$ FORJADO DE H.A. VIGUETAS "IN SITU" PLANTA BAJA

Figura 4. Soluciones constructivas de suelo en los 21 CUI. 


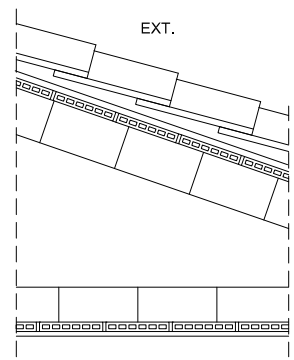

INT.

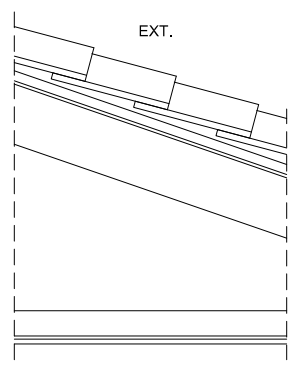

INT

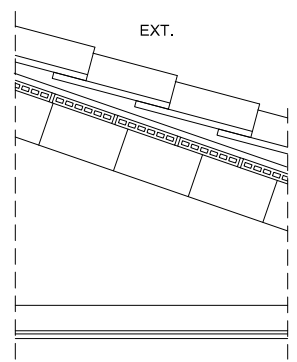

INT

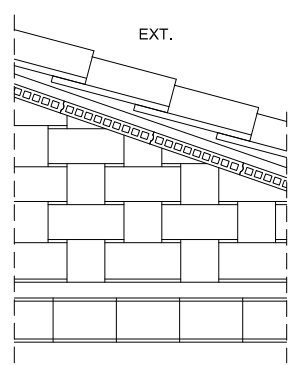

INT

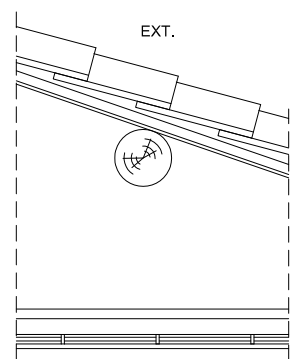

CUBIERTA TIPO C.1

EXTERIOR

TEJA CERÁMICA CURVA TIPO ÁRABE

FORJADO DE VIGUETAS CERÁMICAS ARMADAS

CAPA DE COMPRESIÓN DE HORMIGÓN $\mathrm{e}=2 \mathrm{~cm}$

TABLERO DE RASILLA $\mathrm{e}=3 \mathrm{~cm}$

CÁMARA DE AIRE MUY VENTILADA

FORJADO DE VIGUETAS CERÁMICAS ARMADAS

TABLERO DE RASILLA $\mathrm{e}=3 \mathrm{~cm}$

GUARNECIDO Y ENLUCIDO DE YESO $e=1.5 \mathrm{~cm}$

INTERIOR

\section{CUBIERTA TIPO C.2}

EXTERIOR

TEJA CERÁMICA CURVA TIPO ÁRABE

FORJADO DE ROLLIZOS DE MADERA

TORTA DE BARRO

CANIZO

CÁMARA DE AIRE NO VENTILADA

COSIRONES DE MADERA ATIRANTADOS A LA CUBIERTA

TEJIDO DE CAÑA

GUARNECIDO Y ENLUCIDO DE YESO $e=1.5 \mathrm{~cm}$

INTERIOR
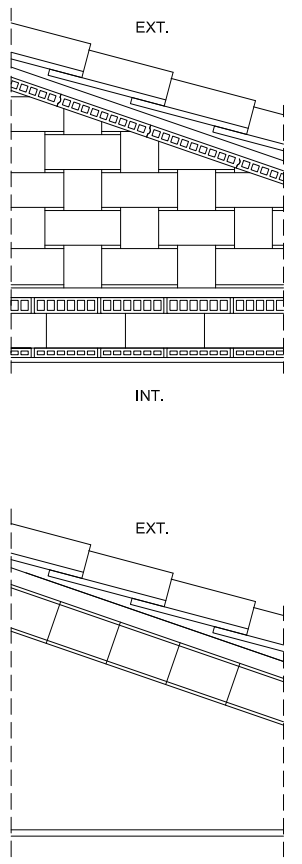

INT

\section{CUBIERTA TIPO C.3}

EXTERIOR

TEJA CERÁMICA CURVA TIPO ÁRABE

FORJADO DE VIGUETAS CERÁMICAS ARMADAS

CAPA DE COMPRESIÓN DE HORMIGÓN e $=2 \mathrm{~cm}$

TABLERO DE RASILLA $\mathrm{e}=3 \mathrm{~cm}$

CÁMARA DE AIRE NO VENTILADA

COSIRONES DE MADERA ATIRANTADOS A LA CUBIERTA

TEJIDO DE CAÑA

GUARNECIDO Y ENLUCIDO DE YESO $\mathrm{e}=1.5 \mathrm{~cm}$

INTERIOR

CUBIERTA TIPO C.4

EXTERIOR

TEJA CERÁMICA CURVA TIPO ÁRABE

CAPA DE COMPRESIÓN DE HORIGÓN $\mathrm{e}=3 \mathrm{~cm}$

TABLERO CERÁMICO MACHIHEMBRADO $\mathrm{e}=4 \mathrm{~cm}$

CÁMARA DE AIRE MUY VENTILADA

FORJADO DE HORMIGÓN ARMADO.

GUARNECIDO Y ENLUCIDO DE YESO e $=1.5 \mathrm{~cm}$

INTERIOR

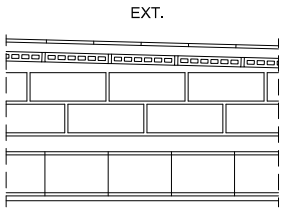

INT.

\section{CUBIERTA TIPO C.8}

EXTERIOR

PLAQUETA CERÁMICA ROJA

CAPA DE COMPRESIÓN DE HORIGÓN $e=3 \mathrm{~cm}$

TABLERO DE RASILLA $e=3 \mathrm{~cm}$

CAMARA DE AIRE MUY VENTILADA

CAPA DE COMPRESIÓN DE HORMIGÓN $\mathrm{e}=5 \mathrm{~cm}$

FORJADO DE VIGUETAS METÁLICAS

GUARNECIDO Y ENLUCIDO DE YESO $e=1.5 \mathrm{~cm}$

INTERIOR

\section{CUBIERTA TIPO C.9}

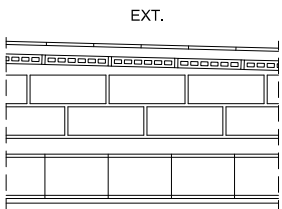

INT.
EXTERIOR

PLAQUETA CERÁMICA ROJA

CAPA DE COMPRESIÓN DE HORIGÓN $\mathrm{e}=3 \mathrm{~cm}$

TABLERO DE RASILLA $e=3 \mathrm{~cm}$

CÁMARA DE AIRE MUY VENTILADA

CAPA DE COMPRESIÓN DE HORMIGÓN e $=5 \mathrm{~cm}$

FORJADO DE VIGUETAS HORMIGÓN
GUARNECIDO Y ENLUCIDO DE YESO $e=1.5 \mathrm{~cm}$

\section{CUBIERTA TIPO C.5}

EXTERIOR

$$
\begin{aligned}
& \text { TEJA CERAMICA CURVA TIPO ARABE } \\
& \text { FORJADO DE ROLLIZOS DE MADERA } \\
& \text { TORTA DE BARRO } \\
& \text { CAÑIZO } \\
& \text { CAMARA DE AIRE NO VENTILADA } \\
& \text { BÓVEDA TABICADA } \\
& \text { RELLENO DE MORTERO e }=3 \mathrm{~cm} \\
& \text { CAMISA DE HORMIGÓN }=5 \mathrm{~cm} \\
& \text { RASILLA CERAMICA e }=3 \mathrm{~cm} \\
& \text { GUARNECIDO Y ENLUCIDO DE YESO } \mathrm{e}=1.5 \mathrm{~cm}
\end{aligned}
$$

INTERIOR

Figura 5. Soluciones constructivas de cubierta en los 21 CUI. 
S.2c.M

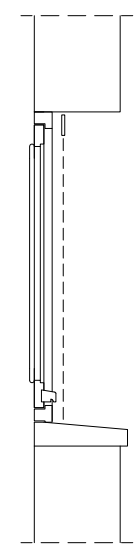

CARPINTERIAA ORIGINAL: MADERAC

PERSIANA DE CADENILLA

S.1.C

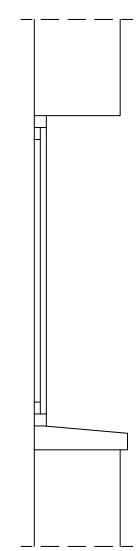

SUSTITUCIÓN: ALUMINIO CORREDERA

S.1.A

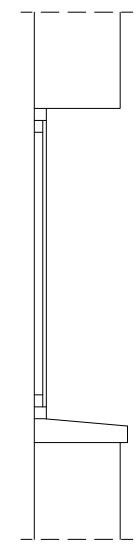

SUSTITUCIÓN ALUMINIO ABATIBLE
S.2b.M

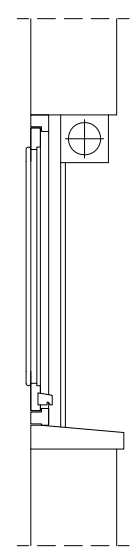

CARPINTERIA ORIGINAL: MADERA CON POSTIGOS

INCORPORACIÓN: CAJÓN DE PERSIANA ADOSADO

S.2c.C

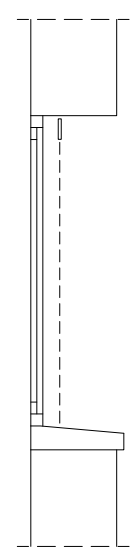

SUSTITUCIÓN: ALUMINIO CORREDERA PERSIANA DE CADENILLA

S.2c.A

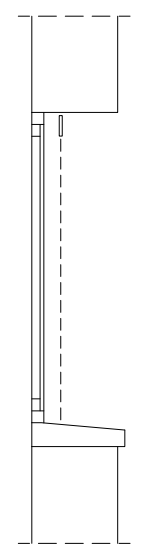

D.3.M

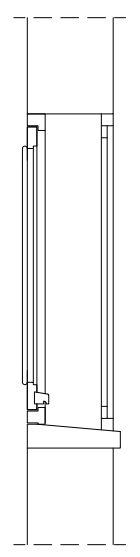

CARPINTERIA ORIGINAL: MADERA CON POSTIGOS

INCORPORACIÓN: CONTRAVENTANA ALUMINIO CORREDERA

S.2b.C

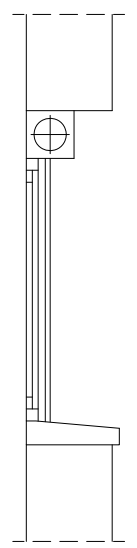

SUSTITUCIÓN: ALUMINIO CORREDERA CON CAJÓN DE PERSIANA

S.2b.A

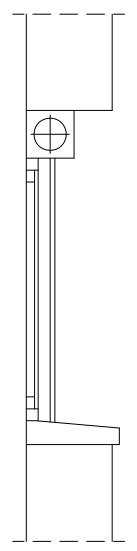

D.4a.M

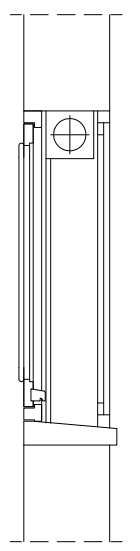

CARPINTERIA ORIGINAL: MADERA CON POSTIGOS

INCORPORACIÓN: CAJÓN DE PERSIANA $Y$ CONTRAVENTANA

S.2a.C

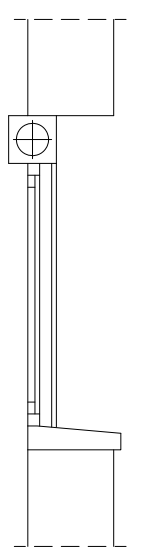

SUSTITUCIÓN: ALUMINIO CORREDERA CON CAJON DE PERSIAN

S.2a.A

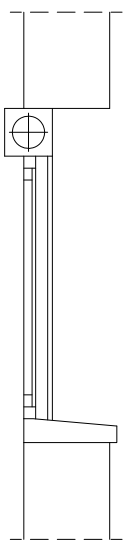

D. 4 b.M

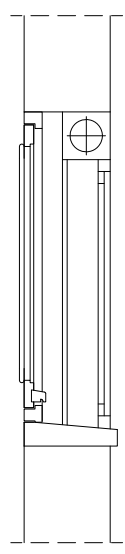

CARPINTERIA ORIGINAL: MADERA CON POSTIGOS

INCORPORACIÓN:

CAJÓN DE PERSIANA Y CONTRAVENTANA AJUSTADA A CAJÓN

\section{D.4a.C}

D.4b.C
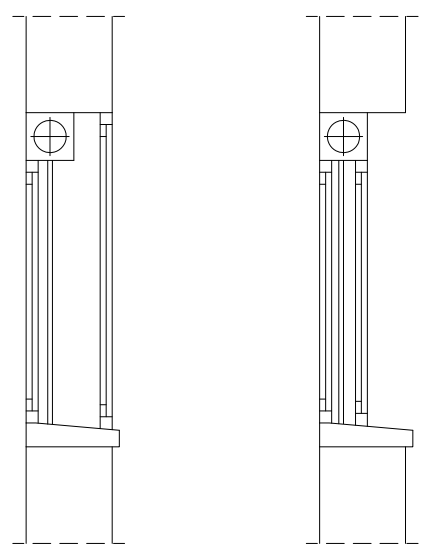

SUSTITUCIÓN: ALUMINIO CORREDERA CON CAJÓN DE PERSIANA

SUSTITUCIÓN: ALUMINIO CORREDERA CON CAJON DE PERSIANAY A

D.4a.A

$\mathrm{H}$
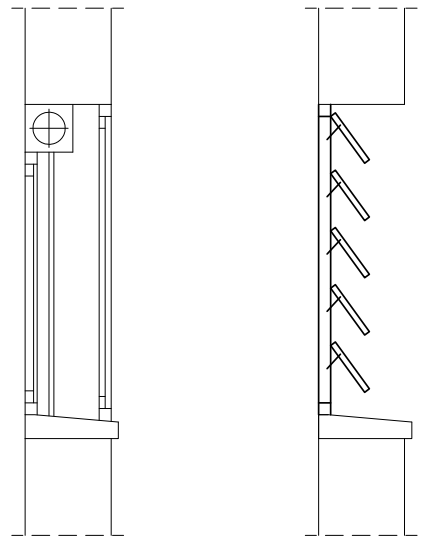

SUSTITUCIÓN: SUSTITUCIÓN: ABATIBLE AINIO ABATIBIE CON CAJÓN DE PERSIANA

Figura 6. Soluciones de huecos de ventana encontrados en los 21 CUI. 
Tabla 1. Porcentaje de los tipos de ventana encontrados en los 21 CUI.

\begin{tabular}{|c|c|c|c|c|c|c|c|c|c|c|c|c|c|}
\hline \multirow[b]{2}{*}{$\begin{array}{l}\text { Tipo de } \\
\text { hueco }\end{array}$} & \multirow[b]{2}{*}{$\begin{array}{c}\text { No } \\
\text { Uds. }\end{array}$} & \multirow[b]{2}{*}{$\begin{array}{c}\% \\
\text { Uds. }\end{array}$} & \multicolumn{3}{|c|}{ tipo de vidrio } & \multicolumn{8}{|c|}{ color carpintería } \\
\hline & & & sencillo & doble & desc. & blanco & $\underset{\text { nio }}{\text { alumi- }}$ & bronce & madera & gris & verde & grafito & desc. \\
\hline S.1.C & 184 & $6,89 \%$ & $71,74 \%$ & $15,22 \%$ & $13,04 \%$ & $18,48 \%$ & $72,83 \%$ & $6,52 \%$ & $0,00 \%$ & $1,63 \%$ & $0,00 \%$ & $0,00 \%$ & $0,54 \%$ \\
\hline S.1.A & 54 & $2,02 \%$ & $29,63 \%$ & $55,56 \%$ & $14,81 \%$ & $59,26 \%$ & $25,93 \%$ & $9,26 \%$ & $5,56 \%$ & $0,00 \%$ & & $0,00 \%$ & $0,00 \%$ \\
\hline S.2a.A & 270 & $10,10 \%$ & $2,22 \%$ & $94,81 \%$ & $2,96 \%$ & $77,04 \%$ & $4,44 \%$ & $9,26 \%$ & $8,52 \%$ & $0,00 \%$ & & $0,00 \%$ & $0,74 \%$ \\
\hline S.2a.C & 404 & $15,12 \%$ & $13,12 \%$ & $78,47 \%$ & $8,17 \%$ & $72,28 \%$ & $14,11 \%$ & $7,43 \%$ & $3,96 \%$ & $0,00 \%$ & $0,00 \%$ & $1,24 \%$ & $0,99 \%$ \\
\hline S.2a.d & 64 & $2,40 \%$ & \multicolumn{3}{|c|}{ desconocido } & \multicolumn{8}{|c|}{ desconocido } \\
\hline S.2b.M & 60 & $2,25 \%$ & $100,00 \%$ & $0,00 \%$ & $0,00 \%$ & $36,67 \%$ & $0,00 \%$ & $0,00 \%$ & $33,33 \%$ & $3,33 \%$ & $23,33 \%$ & $0,00 \%$ & $3,33 \%$ \\
\hline S.2b.C & 688 & $25,75 \%$ & $82,85 \%$ & $10,61 \%$ & $6,54 \%$ & $12,65 \%$ & $81,98 \%$ & $3,92 \%$ & $1,02 \%$ & $0,44 \%$ & $0,00 \%$ & $0,00 \%$ & $0,00 \%$ \\
\hline S.2b.A & 70 & $2,62 \%$ & $34,29 \%$ & $44,29 \%$ & $21,43 \%$ & $54,29 \%$ & $41,43 \%$ & $4,29 \%$ & $0,00 \%$ & $0,00 \%$ & $0,00 \%$ & $0,00 \%$ & $0,00 \%$ \\
\hline S.2b.d & 161 & $6,03 \%$ & \multicolumn{3}{|c|}{ desconocido } & \multicolumn{8}{|c|}{ desconocido } \\
\hline S.2c.M & 251 & $9,39 \%$ & $100,00 \%$ & $0,00 \%$ & $0,00 \%$ & $36,65 \%$ & $0,00 \%$ & $0,00 \%$ & $38,65 \%$ & $9,56 \%$ & $14,74 \%$ & $0,00 \%$ & $0,40 \%$ \\
\hline S.2c.C & 66 & $2,47 \%$ & $95,45 \%$ & $0,00 \%$ & $4,55 \%$ & $3,03 \%$ & $95,45 \%$ & $1,52 \%$ & $0,00 \%$ & $0,00 \%$ & $0,00 \%$ & $0,00 \%$ & $0,00 \%$ \\
\hline S.2c.A & 7 & $0,26 \%$ & $71,43 \%$ & $14,29 \%$ & $14,29 \%$ & $14,29 \%$ & $71,43 \%$ & $14,29 \%$ & $0,00 \%$ & $0,00 \%$ & $0,00 \%$ & $0,00 \%$ & $0,00 \%$ \\
\hline S.2c.d & 78 & $2,92 \%$ & \multicolumn{3}{|c|}{ desconocido } & \multicolumn{8}{|c|}{ desconocido } \\
\hline D.3.M & 8 & $0,30 \%$ & \multicolumn{3}{|c|}{ Interior y exterior sencillos } & $0,00 \%$ & $75,00 \%$ & $0,00 \%$ & $0,00 \%$ & $0,00 \%$ & $0,00 \%$ & $0,00 \%$ & $25,00 \%$ \\
\hline D.3.d & 6 & $0,22 \%$ & \multicolumn{3}{|c|}{ desconocido } & $16,67 \%$ & $50,00 \%$ & $0,00 \%$ & $0,00 \%$ & $0,00 \%$ & $0,00 \%$ & $0,00 \%$ & $33,33 \%$ \\
\hline D.4a.M & 17 & $0,64 \%$ & \multicolumn{3}{|c|}{ Interior y exterior sencillos } & $23,53 \%$ & $76,47 \%$ & $0,00 \%$ & $0,00 \%$ & $0,00 \%$ & $0,00 \%$ & $0,00 \%$ & $0,00 \%$ \\
\hline D.4a.C & 80 & $2,99 \%$ & \multirow{2}{*}{\multicolumn{3}{|c|}{$\begin{array}{l}\text { Interior desconocido y exterior } \\
\text { sencillo }\end{array}$}} & $7,50 \%$ & $80,00 \%$ & $3,75 \%$ & $0,00 \%$ & $0,00 \%$ & $0,00 \%$ & $0,00 \%$ & $8,75 \%$ \\
\hline D.4a.A & 2 & $0,07 \%$ & & & & $0,00 \%$ & $0,00 \%$ & $0,00 \%$ & $0,00 \%$ & $0,00 \%$ & $0,00 \%$ & $0,00 \%$ & $0,00 \%$ \\
\hline D.4a.d & 93 & $3,48 \%$ & \multicolumn{3}{|c|}{ desconocido } & $15,05 \%$ & $70,97 \%$ & $0,00 \%$ & $0,00 \%$ & $0,00 \%$ & $0,00 \%$ & $0,00 \%$ & $13,98 \%$ \\
\hline D.4b.M & 4 & $0,15 \%$ & \multicolumn{3}{|c|}{ Interior y exterior sencillos } & $0,00 \%$ & $100,00 \%$ & $0,00 \%$ & $0,00 \%$ & $0,00 \%$ & $0,00 \%$ & $0,00 \%$ & $0,00 \%$ \\
\hline D. 4 b.C & 41 & $1,53 \%$ & \multicolumn{3}{|c|}{$\begin{array}{l}\text { Interior desconocido y exterior } \\
\text { sencillo }\end{array}$} & $14,63 \%$ & $43,90 \%$ & $9,76 \%$ & $0,00 \%$ & $0,00 \%$ & o,00\% & o,oo\% & $31,71 \%$ \\
\hline $\mathrm{H}$ & 4 & $0,15 \%$ & \multicolumn{3}{|c|}{ No es de aplicación } & $0,00 \%$ & $100,00 \%$ & $0,00 \%$ & $0,00 \%$ & $0,00 \%$ & $0,00 \%$ & $0,00 \%$ & $0,00 \%$ \\
\hline $\mathrm{d}$ & 60 & $2,25 \%$ & \multicolumn{3}{|c|}{ desconocido } & \multicolumn{8}{|c|}{ desconocido } \\
\hline Total & 2672 & $100,00 \%$ & & & & & & & & & & & \\
\hline
\end{tabular}

- Corredera de aluminio (S.2a.C)

- Abatible de aluminio (S.2a.A)

- Con sistema de cierre no accesible a la vista (S.2a.d)

- Con cajón exterior (S.2b)

- Abatible de madera original (S.2b.M)

- Corredera de aluminio (S.2b.C)

- Abatible de aluminio (S.2b.A)

- Con sistema de cierre no accesible a la vista (S.2b.d)

- Persiana de cadenilla

- Abatible de madera originales (S.2c.M)

- Corredera de aluminio (S.2c.C)

- Abatible de aluminio (S.2c.A)

- Con sistema de cierre no accesible a la vista (S.2c.d)

- Ventana doble

- Sin sombreamiento intermedio

- Con ventana interior abatible de madera original (D.3.M)

- Con ventana interior desconocida (D.3.d)

- Con sombreamiento intermedio por medio de persiana enrollable

- Con contraventana a haz exterior corredera

- Con ventana interior de madera original (D.4a.M)

- Con ventana interior corredera de aluminio (D.4a.C)

- Con ventana interior abatible de aluminio (D.4a.A)

- Con ventana interior desconocida (D.4a.d)

- Con contraventana ajustada a cajón de persiana corredera

- Con ventana interior de madera original (D.4b.M)

- Con ventana interior corredera de aluminio (D.4b.C)

- Ventana tipo Hervent $(\mathrm{H})$

- Desconocida (d)
En la Figura 6 las ventanas se ordenan según el material de carpintería. El porcentaje de cada uno de los tipos encontrado queda recogido en la Tabla 2. De esta tabla se deduce que el $45 \%$ de las ventanas son sencillas de vidrio sencillo, de entre las que en torno al $27 \%$ son las originales de madera con más de 50 años representando el $12 \%$ del total aproximadamente. Asimismo se observa que el $37 \%$ son ventanas sencillas con unidades de vidrio aislante y el $18 \%$ son ventanas dobles con la ventana exterior de vidrio sencillo. Se analizan más características de las ventanas en las siguientes secciones.

\subsection{Valoración higrotérmica y acústica de los elementos opacos de la envolvente}

En las Figuras 7, 8 y 9 se observa el porcentaje respecto el total en los 21 conjuntos de las soluciones constructivas de la envolvente utilizadas, de fachada, cubiertas y suelos respectivamente, y su nivel de cumplimiento respecto a la normativa actual mediante gráfico de barras.

Según el DB-HE1, en las obras de reforma en las que se renueve más del $25 \%$ de la superficie total de la envolvente térmica final del edificio, como serán las rehabilitaciones a las que convendría someter estos edificios de viviendas, se limitará la demanda energética conjunta del edificio de manera que sea inferior a la del edificio de referencia. Éste, según el CTE, es un edificio obtenido a partir del edificio objeto, con su misma forma, tamaño, orientación, zonificación interior, uso de cada espacio, e iguales obstáculos remotos, y unas soluciones constructivas tipificadas, cuyos parámetros característicos deben cumplir con una transmitancia límite $\left(\mathrm{U}_{\mathrm{lim}}\right)$ 
Tabla 2. Exigencias acústicas en los CUI en función del mayor $\mathrm{L}_{\mathrm{d}}$ del conjunto.

\begin{tabular}{|c|c|c|c|c|c|c|c|c|c|}
\hline \multirow[b]{2}{*}{ Conjuntos de Interés } & \multicolumn{5}{|c|}{$L_{d}(d b(A))$} & \multicolumn{4}{|c|}{ Exigencias (dB(A)) } \\
\hline & $55-60$ & $60-65$ & $65-70$ & $70-75$ & $>75$ & $\begin{array}{c}\mathrm{D}_{\text {2m,nT,Atr }} \\
\text { dormitorios }\end{array}$ & $\underset{\text { cubiertas }}{\mathbf{R}_{\mathrm{A}, \mathrm{tr}}}$ & $\underset{\text { parte ciega fachadas }}{\mathbf{R}_{\mathrm{A}, \mathrm{tr}}}$ & $\underset{\text { huecos }}{\mathbf{R}_{\mathrm{A}, \mathrm{tr}}}$ \\
\hline \multirow{2}{*}{ Aloy Sala } & & & & & & \multirow{4}{*}{47} & \multirow{4}{*}{49} & 55 & 47 \\
\hline & & & & & & & & 60 & 47 \\
\hline \multirow{2}{*}{$\begin{array}{l}\text { Casta Álvarez } \\
\text { Fray Julián Garcés } \\
\text { Balsas de Ebro Viejo } \\
\text { Parcelación Daman } \\
\text { Alférez Rojas } \\
\text { San Jorge }\end{array}$} & & & & & & & & 55 & 45 \\
\hline & & & & & & & & 60 & 44 \\
\hline \multirow{3}{*}{$\begin{array}{l}\text { Girón } \\
\text { Vizconde de Escoriaza } \\
\text { Santa Rosa } \\
\text { Puente Virrey-Tejar } \\
\text { Francisco Franco } \\
\text { General Urrutia } \\
\text { Arzobispo Doménech }\end{array}$} & & & & & & \multirow{3}{*}{42} & \multirow{3}{*}{44} & 50 & 40 \\
\hline & & & & & & & & 55 & 39 \\
\hline & & & & & & & & 60 & 39 \\
\hline \multirow{3}{*}{$\begin{array}{l}\text { Venecia } \\
\text { Torrero } 1^{\text {a }} \text { fase } \\
\text { Agustín Gericó } \\
\text { Puente Virrey-Rosellón }\end{array}$} & & & & & & \multirow{3}{*}{37} & \multirow{6}{*}{39} & 40 & 37 \\
\hline & & & & & & & & 45 & 35 \\
\hline & & & & & & & & 50 & 34 \\
\hline \multirow{3}{*}{ Ortiz Zárate } & & & & & & \multirow{3}{*}{37} & & 40 & 35 \\
\hline & & & & & & & & 45 & 32 \\
\hline & & & & & & & & 50 & 31 \\
\hline \multirow{3}{*}{ Teniente Polanco } & & & & & & \multirow{3}{*}{32} & \multirow{3}{*}{35} & 35 & 32 \\
\hline & & & & & & & & 40 & 30 \\
\hline & & & & & & & & 45 & 29 \\
\hline \multirow{3}{*}{ Casetas } & & & & & & \multirow{3}{*}{30} & \multirow{3}{*}{33} & 35 & 29 \\
\hline & & & & & & & & 40 & 28 \\
\hline & & & & & & & & 45 & 28 \\
\hline
\end{tabular}

que depende de la zona climática, siendo en este caso la D3 correspondiente a Zaragoza.

Según el DB-HR para proteger al edificio frente al ruido procedente del exterior, el aislamiento acústico a ruido aéreo de los elementos de la envolvente, $\mathrm{D}_{2 \mathrm{~m}, \mathrm{nT} \text {,Atr }}$, entre un recinto protegido y el exterior no será menor que unos ciertos valores, en función del uso del recinto (dormitorios o estancias) y de los valores del índice de ruido día, $\mathrm{L}_{\mathrm{d}}$, según el mapa de ruido de Zaragoza (28). Los valores de $\mathrm{L}_{d}$ en el entorno de los bloques de vivienda de los 21 CUI se reflejan en la Tabla 2, así como el $\mathrm{D}_{2 \mathrm{~m}, \mathrm{nT} \text {,Atr }}$ correspondiente al máximo valor de $\mathrm{L}_{\mathrm{d}} \mathrm{y}$ al uso dormitorios. Siguiendo la opción simplificada del DB-HR, los valores mínimos que deben cumplir los elementos de la envolvente dependen de $\mathrm{D}_{2 \mathrm{~m}, \mathrm{nT} \text {,Atr }} \mathrm{y}$ del porcentaje de huecos. Los huecos representan entre el $17 \mathrm{y}$ el $23 \%$ de las fachadas en todos los CUI, excepto en las torres de Aloy Sala con un $35 \%$, y en el conjunto Ortiz Zárate con un $14 \%$. De esta forma, los valores de $\mathrm{R}_{\mathrm{A}, \mathrm{tr}}$ exigidos en cubiertas, parte ciega de fachadas y huecos son los recogidos en la Tabla 2. Las propiedades de los materiales para la predicción del $\mathrm{R}_{\mathrm{A}, \mathrm{tr}}$ de las soluciones constructivas opacas quedan recogidas en la Tabla 3.

La altura de las barras en las Figuras 7, 8 y 9 representa el índice de reducción acústica estimado (barras superiores) o la transmitancia del elemento calculada (barras inferiores), y la anchura de las barras representa el porcentaje que representa dicho tipo de solución de la envolvente respecto del total. Los límites establecidos por la normativa se representan en líneas horizontales: la transmitancia límite máxima y el valor más alto para cada conjunto del índice de reducción acústico mínimo. Adicionalmente en el diagrama de barras de la transmitancia, un eje a la derecha representa el espesor de aislamiento que sería suficiente añadir para cumplir los requerimientos normativos $\left(\mathrm{U}_{\mathrm{lim}}\right)$, calculado para un aislamiento con conductividad media de $0,04 \mathrm{~W} / \mathrm{K} \cdot \mathrm{m}$. En la parte inferior de las Figuras se recogen los nombres de los conjuntos y su año de proyecto y construcción y las soluciones constructivas empleadas, ordenadas cronológicamente. Adicionalmente en la Figura 6 se señalan las soluciones estructurales.

Como se puede observar, todos los cerramientos existentes tienen una transmitancia superior a los valores límite del edificio de referencia. El $20 \%$ de las fachadas presentan una transmitancia unas tres veces superior al valor límite requiriendo 4-4,5 cm de aislamiento. Para el resto de fachadas es suficiente con 2-4 cm. La solución de forjado sanitario F.S.1, que representa el $4 \%$ de suelos requiere $6-6,5 \mathrm{~cm}$, mientras que los demás tipos de suelo requieren $2-4 \mathrm{~cm}$. Las cubiertas presentan una transmitancia entre 3 y 7 veces mayores que el valor límite requerido, lo que implica la necesidad de disponer aislamientos térmicos de 7-9 cm.

Se observa en la Figura 7 que las soluciones de fachada con un pie y medio de ladrillo macizo o con un pie de ladrillo macizo más trasdosado o de adobe (con una representación en torno al $45 \%$ ) son aptas acústicamente para cualquier nivel de $\mathrm{L}_{\mathrm{d}}$ presente en los conjuntos estudiados. Las fachadas de 1 pie de ladrillo macizo o de medio pie más trasdosado o de bloques de hormigón macizo (en torno al 33 \%) presentan buenas prestaciones acústicas, aunque no son aptas en algunos conjuntos donde los niveles de $\mathrm{L}_{\mathrm{d}}$ son elevados. Las restantes 


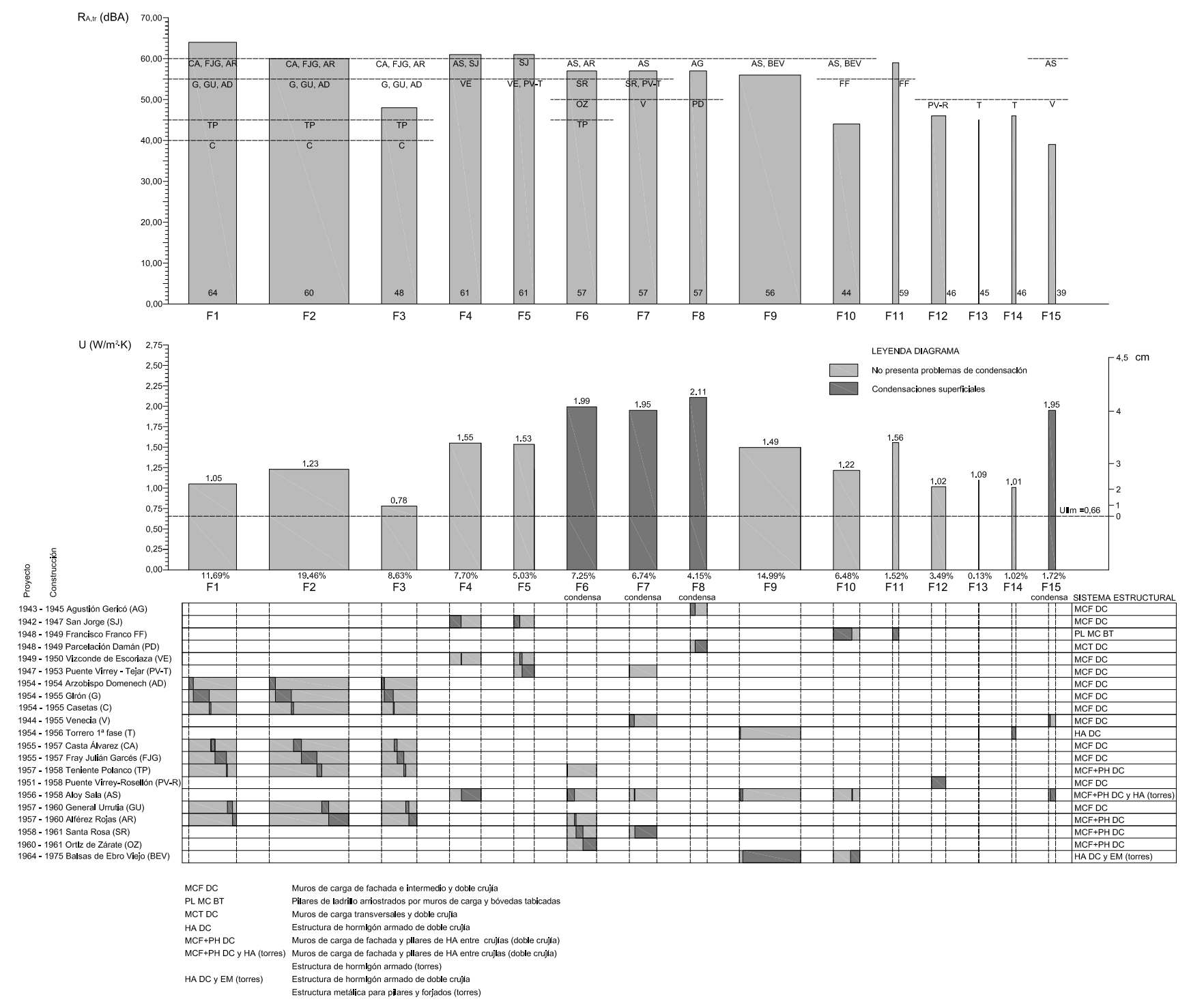

Figura 7. Análisis de las soluciones constructivas de fachada utilizadas.

Tabla 3. Propiedades de los materiales considerados para la predicción del $\mathrm{R}_{\mathrm{A}, \mathrm{tr}}$ de las soluciones constructivas (entre paréntesis: fuente de los datos).

\begin{tabular}{|l|c|c|}
\hline \multicolumn{1}{|c|}{ Material } & Densidad (kg/m $\left.{ }^{3}\right)$ & Módulo de Young (GPa) \\
\hline Ladrillo hueco cerámico & $770(22)$ & $4(29)$ \\
\hline Ladrillo perforado & $780(22)$ & $4(29)$ \\
\hline Ladrillo macizo & $2300(22)$ & $4(29)$ \\
\hline Mortero & $1000(22)$ & $11(30)$ \\
\hline Enlucido & $690(25)$ & $2,01(25)$ \\
\hline Rasillas & $1600(25)$ & $8,9(25)$ \\
\hline Torta de barro o adobe & $1770(22)$ & $2,54(31)$ \\
\hline Teja & $2392(25)$ & $11,75(25)$ \\
\hline Hormigón & $2340(25)$ & $11,00(25)$ \\
\hline Bloque de hormigón & $1905(25)$ & $15,02(25)$ \\
\hline
\end{tabular}

fachadas de ladrillo hueco de diferentes espesores (en torno al $22 \%$ ) presentan problemas acústicos en todos los conjuntos en los que se usan.

Asimismo se observa en la Figura 8, que las cubiertas sin cámara de aire ventilada cumplen holgadamente los actuales estándares acústicos. Para las cubiertas con cámara de aire ventilada el método predictivo empleado no es lo suficientemente preciso.

En el estudio cronológico de las soluciones, se observa que a partir del año 1956 aparece junto con la ampliamente utilizada tipología de vivienda social de planta baja más 3 ó 4 plantas la tipología torre de entre 8 y 12 alturas en la que la facha- 


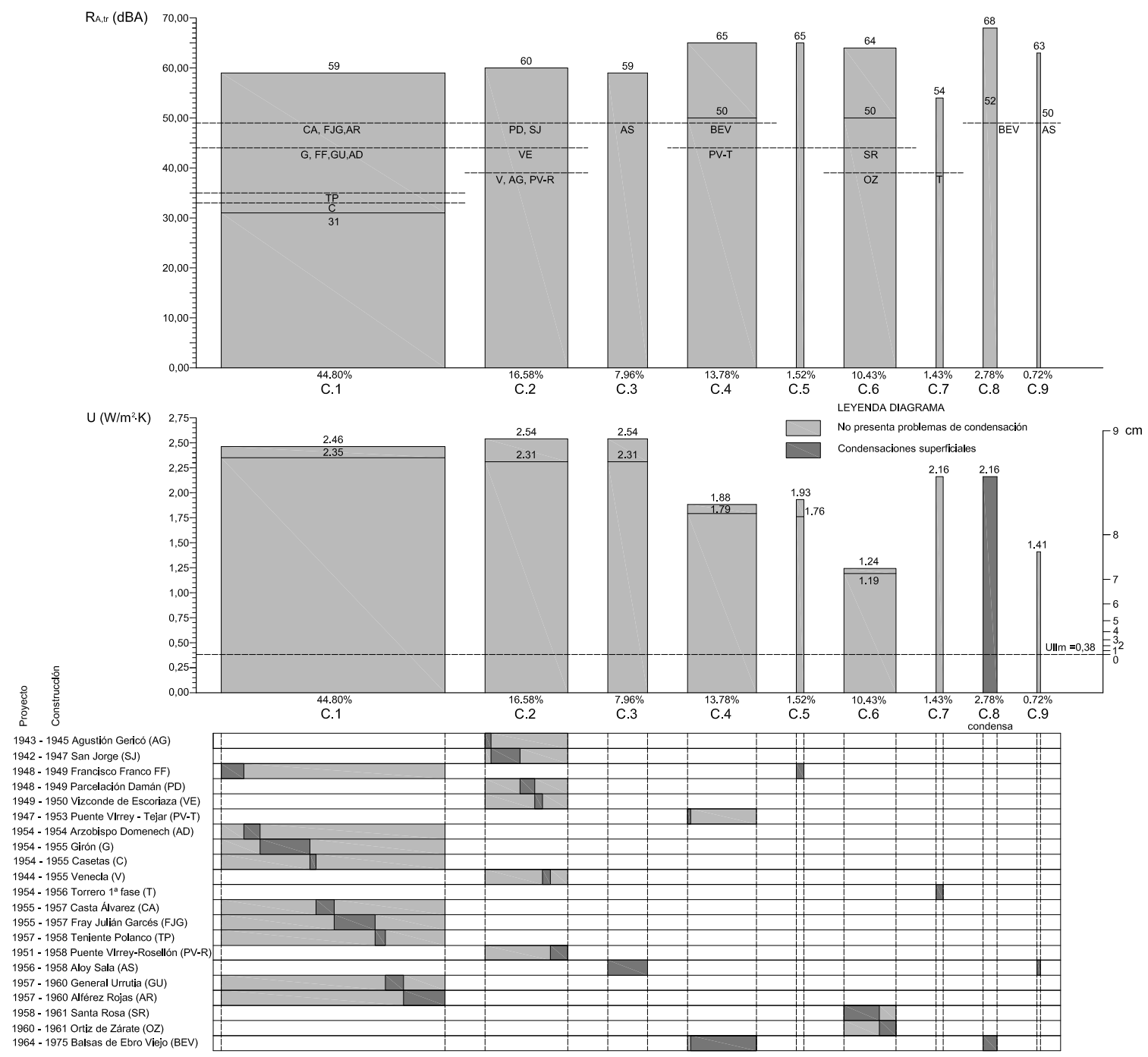

Figura 8. Análisis de las soluciones constructivas de cubierta utilizadas.

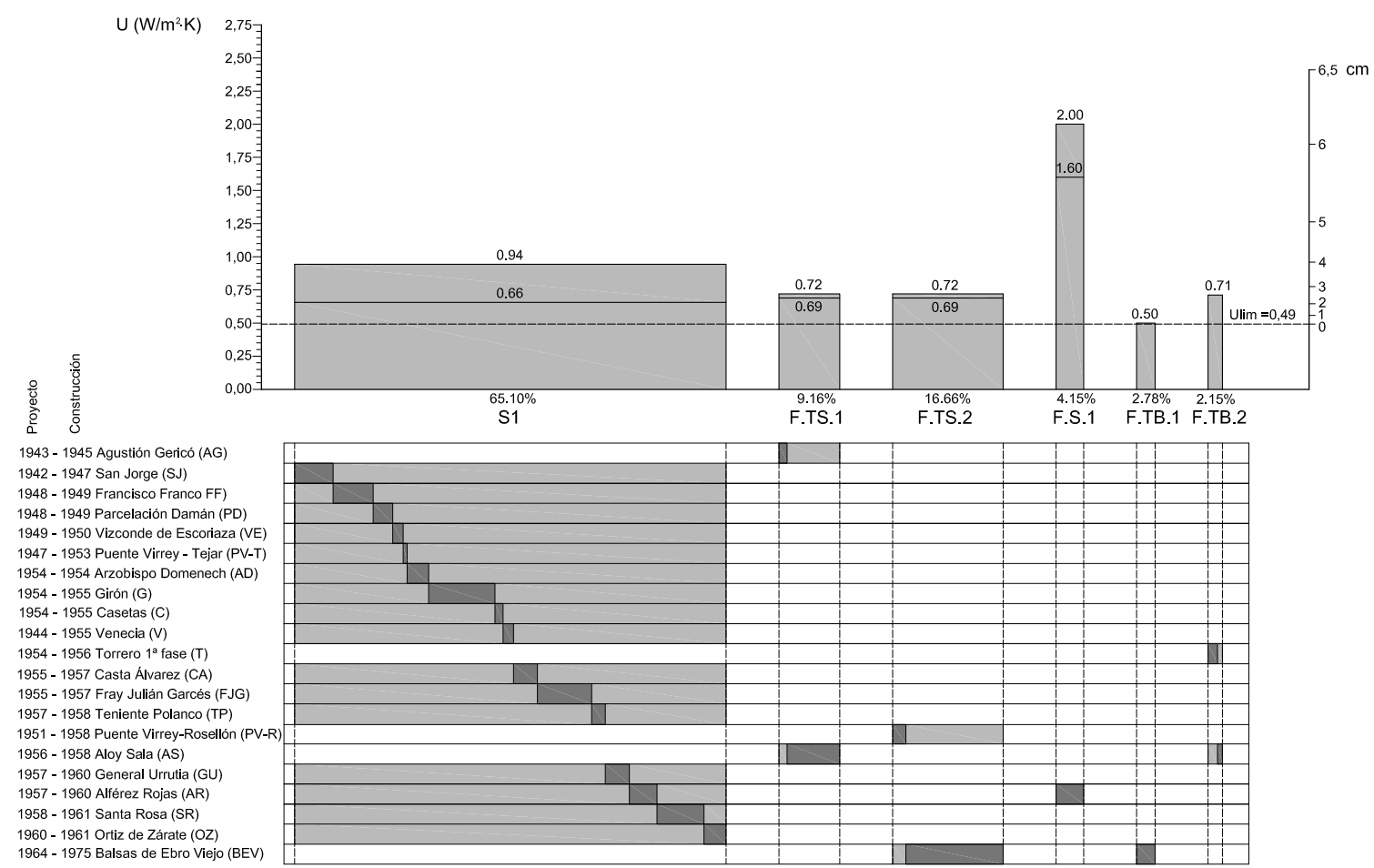

Figura 9. Análisis de las soluciones constructivas de suelos utilizadas. 
Tabla 4. Caracterización del comportamiento higrotérmico de los huecos (leyenda: CP: cajón de persiana; cond. sup.: condensaciones superficiales).

\begin{tabular}{|c|c|c|}
\hline Tipo & Vidrio sencillo & Vidrio doble \\
\hline S.1.C & \multirow{2}{*}{$\begin{array}{l}\mathrm{U}_{\text {vidriomarec }}=5,7 \mathrm{~W} / \mathrm{m}^{2} \cdot \mathrm{K} \\
\operatorname{Sin~CP}\end{array}$} & \multirow{2}{*}{$\begin{array}{l}\mathrm{U}_{\text {vidrio+marco }}=3,4-4,2 \mathrm{~W} / \mathrm{m}^{2} \cdot \mathrm{K} \\
\text { Sin CP }\end{array}$} \\
\hline S.1.A & & \\
\hline S.2a.C & \multirow{2}{*}{$\begin{array}{l}\mathrm{U}_{\text {vidriotmarce }}=5,7 \mathrm{~W} / \mathrm{m}^{2} \cdot \mathrm{K} \\
\text { Puente térmico de CP } \\
\text { Sin cond. sup. en } \mathrm{CP}\end{array}$} & \multirow{2}{*}{$\begin{array}{l}\mathrm{U}_{\text {vidriotmarco }}=3,4-4,2 \mathrm{~W} / \mathrm{m}^{2} \cdot \mathrm{K} \\
\text { Puente térmico de } \mathrm{CP} \\
\text { Sin cond. sup. en } \mathrm{CP}\end{array}$} \\
\hline S.2a.A & & \\
\hline S.2b.M & $\begin{array}{l}\mathrm{U}_{\text {vidrio+marco }}=4,3-5,0 \mathrm{~W} / \\
\mathrm{m}^{2} \cdot \mathrm{K} \\
\text { Sin puente térmico de } \mathrm{CP}\end{array}$ & - \\
\hline S.2b.C & \multirow{2}{*}{$\begin{array}{l}\mathrm{U}_{\text {vidriomarce }}=5,7 \mathrm{~W} / \mathrm{m}^{2} \cdot \mathrm{K} \\
\text { Puente térmico de CP } \\
\text { Cond. sup. en CP }\end{array}$} & \multirow{2}{*}{$\begin{array}{l}\mathrm{U}_{\text {vidrio+marco }}=3,5-4,2 \mathrm{~W} / \mathrm{m}^{2} \cdot \mathrm{K} \\
\text { Puente térmico de CP } \\
\text { Cond. sup. en CP }\end{array}$} \\
\hline S.2b.A & & \\
\hline S.2c.M & $\begin{array}{l}\mathrm{U}_{\text {vidrio+marco }}=4,3-5,0 \mathrm{~W} / \\
\mathrm{m}^{2} \cdot \mathrm{K} \\
\mathrm{Sin} \mathrm{CP}\end{array}$ & - \\
\hline S.2c.C & \multirow{2}{*}{$\begin{array}{l}\mathrm{U}_{\text {vidrio+marco }}=5,7 \mathrm{~W} / \mathrm{m}^{2} \cdot \mathrm{K} \\
\mathrm{Sin} \mathrm{CP}\end{array}$} & - \\
\hline S.2c.A & & $\begin{array}{l}\mathrm{U}_{\text {vidrio+marco }}=3,4-4,2 \mathrm{~W} / \mathrm{m}^{2} \cdot \mathrm{K} \\
\text { Sin CP }\end{array}$ \\
\hline D.3.M & \multicolumn{2}{|c|}{$\mathrm{U}_{\text {vidrio+marco }}=1,32-1,37, \operatorname{Sin} \mathrm{CP}$} \\
\hline D.4a.M & \multicolumn{2}{|c|}{$\mathrm{U}_{\text {vidrio+marco }}=1,32-1,37$, Sin puente térmico de CP } \\
\hline D.4a.C & \multirow{2}{*}{\multicolumn{2}{|c|}{$\mathrm{U}_{\text {vidrio+marco }}=1,23-1,4 \mathrm{O}$, Puente térmico de $\mathrm{CP}$, Sin cond. sup. en $\mathrm{CP}$}} \\
\hline D.4a.A & & \\
\hline D.4b.M & \multicolumn{2}{|c|}{$\mathrm{U}_{\text {vidrio+marco }}=1,32-1,37$, Puente térmico de CP, Cond. sup. en CP } \\
\hline D.4b.C & \multicolumn{2}{|c|}{$\mathrm{U}_{\text {vidrio+marco }}=1,23-1,40$, Puente térmico de $\mathrm{CP}$, Cond. sup. en CP } \\
\hline $\mathbf{H}$ & \multicolumn{2}{|l|}{ Ventanas no herméticas } \\
\hline
\end{tabular}

da deja de tener función portante disminuyendo su espesor considerablemente con lo que se abandonan las soluciones de fachada con mejor comportamiento acústico.

\subsection{Valoración higrotérmica y acústica de los elementos semitransparentes de la envolvente}

El valor límite de transmitancia para huecos a norte es $3 \mathrm{~W} / \mathrm{m}^{2} \bullet \mathrm{K}$, y para las demás orientaciones es $3,5 \mathrm{~W} / \mathrm{m}^{2} \bullet \mathrm{K}$. Como se observa en la Tabla 4, prácticamente todas las ventanas sencillas se quedan por encima de estos valores (82 \%), y todas las dobles por debajo (18\%). Puentes térmicos de cajón de persiana se producen en todas las soluciones en que el cajón se interpone entre la ventana y el cerramiento opaco (soluciones S.2a.C, S.2a.A, S.2b.C, S.2b.A, D.4a.C, D.4a.A, D.4b.C, D.4b.M), que representan el $72 \%$ de los huecos. Tendremos condensaciones cuando el cajón está expuesto hacia el exterior, es muy ventilado y no lleva aislamiento (soluciones S.2b.C, S2b.A, D.4b.M., D.4b.C), representando el $37 \%$ de las ventanas. Por ello podemos concluir que el sistema envolvente semitransparente es igualmente muy deficiente energéticamente.

La predicción acústica en huecos es particularmente compleja porque depende en gran medida de las infiltraciones que puedan existir, por ejemplo a través de capialzados, por ello se han usado las dos aproximaciones comentadas en la metodología de investigación. Algunas soluciones no se han podido predecir por ausencia de datos y se han consignado como «?» en la Tabla 5. Los intervalos se emplean cuando la predicción depende de variables con diferentes valores, o cuando no se disponen de datos de las variables precisos.

En las ventanas sencillas (82 \%) se observa una falta de aislamiento acústico acusada generalizada. Para las ventanas
Tabla 5. Predicción de $\mathrm{R}_{\mathrm{A}, \mathrm{tr}}$ de huecos según el CEC-CTE (22) y según Moreno y Peña (27).

\begin{tabular}{|c|c|c|}
\hline \multirow{2}{*}{ Tipos de hueco } & \multicolumn{2}{|c|}{$\mathbf{R}_{\mathrm{A}, \mathrm{tr}}(\mathbf{d B A})$} \\
\cline { 2 - 3 } & Según CEC & Moreno y Peña \\
\hline S.1.C & $26-27$ & 19,8 \\
\hline S.1.A & $26-27$ & 21,0 \\
\hline S.2a.A & $?$ & 21,0 \\
\hline S.2a.C & $?$ & 19,8 \\
\hline S.2b.M & 26,0 & $?$ \\
\hline S.2b.C & $?$ & 17,7 \\
\hline S.2b.A & $?$ & 18,3 \\
\hline S.2c.M & 26,0 & $?$ \\
\hline S.2c.C & $26-27$ & 19,8 \\
\hline S.2c.A & $26-27$ & 21,0 \\
\hline D.3.M & $26-?$ & $?$ \\
\hline D.4a.C & $27-44$ & $21,2-?$ \\
\hline D.4a.A & $27-44$ & $?$ \\
\hline D.4b.C & $?$ & $?$ \\
\hline & & \\
\hline
\end{tabular}

dobles (18\%) la predicción es insuficientemente precisa para llegar a conclusiones.

\section{CONCLUSIONES}

Los conjuntos urbanos de viviendas sociales en barrios vulnerables de Zaragoza construidos entre 1945 y 1975 muestran una clara aunque lenta evolución hacia la planta libre, siguiendo los postulados de la arquitectura moderna, por el hecho de que España atraviesa por el periodo de la autarquía y existen importantes restricciones del acero, cemento y el transporte de materiales a obra. Se observa en los bloques de baja más tres o cuatro alturas que en los primeros conjuntos la estructura se resuelve a base de muro de carga de doble crujía, mientras que en años posteriores el muro de carga intermedio se desmaterializa para convertirse en un pórtico de hormigón armado dotando de una mayor flexibilidad en la distribución en planta a la vivienda. Esta primera evolución mantiene el carácter estructural de la envolvente por lo que su comportamiento térmico y acústico no cambia. Por otro lado, aparece de forma más puntual a partir de 1956 la tipología de torres que requiere para su construcción la evolución a la estructura de pilares y forjados unidireccionales donde las fachadas ya no tienen una función estructural, reduciendo los espesores de éstas en detrimento de su aislamiento acústico. Este es el caso de las torres de Aloy Sala y del conjunto Balsas de Ebro Viejo. También se observa esta solución constructiva en el barrio de Torrero debido a la liberalización de la planta baja para albergar uso comercial.

En las viviendas sociales de barrios vulnerables la estimación de los consumos energéticos como forma de análisis de su obsolescencia constructiva no parece acertada debido a la alta incidencia de pobreza energética. Por ello, en este artículo hemos propuesto su análisis a través de la comparativa del comportamiento de los elementos de la envolvente térmica y acústica con los niveles actualmente considerados de confort en la normativa.

Energéticamente, la mayor parte de las viviendas presenta una orientación favorable y posibilidades de ventilación cru- 
zada, como el movimiento modernista postulaba. La parte opaca de la envolvente, sin embargo, está lejos de cumplir los estándares actuales, particularmente en el caso de cubiertas cuya transmitancia es de tres a siete veces superior a los estándares actuales. Los suelos tienen una transmitancia más aceptable gracias al efecto del terreno o de los espacios no habitables bajo ellos, de forma que podría realizarse la intervención en estos edificios sin rehabilitarlos teniendo en cuenta que resulta desaconsejable desalojar a los habitantes de estas viviendas con escasos recursos económicos. Únicamente la solución de forjado sanitario en Alférez Rojas requeriría plantearse la posibilidad de aislar el suelo. Las ventanas dobles encontradas cumplen con la normativa actual y las ventanas sencillas no, por lo que la instalación de contraventanas resulta de interés, garantizando que el cajón de persiana no dé al exterior sin aislamiento para evitar las condensaciones superficiales en el mismo.

El análisis acústico implica la consideración simultánea de las soluciones de huecos y parte ciega y el índice de ruido día, $\mathrm{L}_{\mathrm{d}}$. En general, la parte ciega de fachadas con ladrillo macizo -solución más usada- o bloque macizo de hormigón presenta una alta capacidad aislante apta para todos o casi todos los niveles de $\mathrm{L}_{\mathrm{d}}$. Las fachadas de ladrillos huecos no siempre son suficientes, dependiendo de su espesor y $L_{d}$ del emplazamiento. La parte más débil de las fachadas es la semitransparente. Las ventanas sencillas presentes en los 21 CUI -que representan el 82 \% de las ventanas- no cumplen los estándares actuales, siendo las más débiles las que tienen cajón de persiana no prefabricado -en torno al $35 \%$-. Las cubiertas ventiladas (73 \%) y las ventanas dobles (18\%) requieren métodos de predicción más precisos para poder llegar a conclusiones.

\section{AGRADECIMIENTOS}

Agradecemos el apoyo económico recibido del Ministerio de Economía y Competitividad, proyecto BIA2013-44001-R: Protocolo de Diseño Integrado para la Rehabilitación de la Vivienda Social y Regeneración Urbana.

Agradecemos al estudiante de ciclo formativo de grado superior Gonzalo Eynar Miranda Laruta del centro público de formación profesional Corona de Aragón su colaboración en la organización de la información y superficiado de fachadas, cubiertas y plantas.

Agradecemos asimismo a Juan Rubio del Val de la Sociedad Municipal Zaragoza Vivienda facilitarnos información sobre los proyectos de rehabilitación de las viviendas sociales.

\section{REFERENCIAS}

(1) OCDE. (1998). Integrating Distressed Urban Areas, Paris: The Organisation for Economic Co-operation and Development.

(2) European Union. (1997). Agenda 2000. For a stronger and wider Union, Bulletin of the European Union, $\mathrm{n}^{\circ} 5 / 97$.

(3) Arias Goytre, F. (2000). La desigualdad urbana en España, Madrid: Ministerio de Fomento.

(4) Ruiz Palomeque, G., Rubio del Val, J. (2006). Nuevas propuestas de rehabilitación urbana en Zaragoza. Estudio de Conjuntos Urbanos de Interés, Zaragoza: Sociedad Municipal de Rehabilitación Urbana de Zaragoza.

(5) Gobierno de Aragón. (2001). Acuerdo del Consejo de Ordenación del Territorio de 13 de junio de 2001, por el que se aprueba la Revisión del Plan General de Ordenación Urbana de Zaragoza, Boletín Oficial de Aragón, $\mathrm{n}^{0} 71$.

(6) Economidou, M. (Project lead). (2011). Europe's buildings under the microscope. A country-by-country review of the energy performance of buildings. Buildings Performance Institute Europe (BPIE).

(7) WWF España. (2010). Potencial de ahorro energético y de reducción de emisiones de CO2 del parque residencial existente en España en 2020, Madrid: WWF/Adena.

(8) Cuchí, A., Sweatman, P. (2012). INFORME GTR 2012. Una visión-país para el sector de la edificación en España. Plan de acción para un Nuevo Sector de la Vivienda, Madrid: Grupo de Trabajo sobre Rehabilitación, Green Building Council España y Fundación Conama.

(9) Cheng, V., Steemers, K. (2012). Modelling domestic energy consumption at district scale: A tool to support national and local energy policies, Environmental Modelling \& Software, 26: 1186-1198.

(10) Natarajana, S., Padgetb, J., Elliott, L. (2011). Modelling UK domestic energy and carbon emissions: an agent-based approach, Energy and Buildings, 43: 2602-2612, doi: http://dx.doi.org/10.1016/j.enbuild.2011.05.013.

(11) IDAE (2011). Proyecto Sech-Spahousec. Análisis del consumo energético del sector residencial en España, Madrid: Instituto para la Diversificación y Ahorro de Energía.

(12) Guerra, O., Itard, L., Visscher, H. (2009). The effect of occupancy and building characteristics on energy use for space and water heating in Dutch residential stock, Energy and Buildings, 41: 1223-1232, doi: http://dx.doi.org/10.1016/j.enbuild.2009.07.002.

(13) Tirado Herrero, S., Jiménez Meneses, L., López Fernández, J.L., Martín García, J. (2014). Pobreza energética en España. Análisis de tendencias. Madrid: Asociación de Ciencias Ambientales.

(14) López-Mesa, B., Palomero Cámara, J.I., Ortega Zapata, A., del Amo Sancho, A. (2013). La rehabilitación y la mejora de la eficiencia energética de la vivienda social a examen. En: J. Tejedor (Ed.) Rehabilitación y Regeneración Urbana en España. Situación actual y perspectivas. Monografías de la Revista Aragonesa de Administración Pública, XV: $283-319$.

(15) Sendra, J.J., Domínguez-Amarillo, S., Bustamante, P., León, A.L. (2013). Energy intervention in the residential sector in the south of Spain: Current challenges. Informes de la construcción, 65(532): 457-464, doi: http://dx.doi.org/10.3989/ic.13.074.

(16) MFOM. (2010). Análisis urbanístico de Barrios Vulnerables en España. Introducción al Informe General 20o1: Metodología, Estructura del Catálogo y Créditos. Madrid: Ministerio de Fomento (MFOM).

(17) Gobierno de Aragón. (2008). Resolución de 20 de junio de 2008, de la Dirección General de Urbanismo, por la que se da publicidad al Acuerdo adoptado por el Consejo de Ordenación del Territorio, en su reunión celebrada el día 6 de junio de 2008, relativo al Texto Refundido del Plan General de Ordenación Urbana de Zaragoza, Boletín Oficial de Aragón, nº91. 
(18) Sánchez Ventura, J. M. (1948). El problema de la vivienda barata, Zaragoza: El Noticiero.

(19) Martí Nasarre de Letosa, A. (1994). La vivienda obrera en Zaragoza 1939-1947. Cuadernos de Zaragoza, 64:1-138.

(20) Cervero Sánchez, N. (2013). El papel de la rehabilitación integral de barrios en la recuperación de las periferias. Intervención en el grupo «El Picarral», Zaragoza. Revista electrónica de Patrimonio Histórico e-RPH, $\mathrm{n}^{\circ}{ }_{13}$ (diciembre). http://www.revistadepatrimonio.es/revistas/numero13/intervencion/estudios/articulo.php.

(21) MFOM. (2013). Documento de Apoyo al Documento Básico DB-HE Ahorro de energía del Código Técnico de la Edificación DA DB-HE / 1 Cálculo de parámetros característicos de la envolvente. Ministerio de Fomento (MFOM). http:// www.codigotecnico.org/web/recursos/documentos.

(22) IETcc. (2010). Catálogo de Elementos Constructivos del CTE. Instituto Eduardo Torroja de ciencias de la construcción (IETcc). http://www.codigotecnico.org/web/recursos/aplicaciones/contenido/texto_oo12.html .

(23) MFOM. (2013). Documento de Apoyo al Documento Básico DB-HE Ahorro de energía del Código Técnico de la Edificación DA DB-HE / 2 Comprobación de limitación de condensaciones superficiales e intersticiales en los cerramientos. Ministerio de Fomento (MFOM). http://www.codigotecnico.org/web/recursos/documentos/.

(24) Ballagh, K. O. (2004, 22-24 de agosto). Accuracy of Prediction Methods for Sound Transmission Loss. En The 33rd International Congress and Exposition on Noise Control Engineering. Prague.

(25) Insul 7.0.6, Marshall Day Acoustics, New Zealand. http://www.insul.co.nz/features/.

(26) AENOR-CEN. (2007). UNE-EN ISO 6946:2007 Componentes y elementos para la edificación. Resistencia térmica y transmitancia térmica. Método de cálculo. Asociación Española de Normalización (AENOR)

(27) Moreno, A., Peña, M.A. (2002). Estudio de apoyo para el Código Técnico de la Edificación del Ministerio de Fomento. Aislamiento acústico en edificios: valores medidos in situ versus valores de predicción numérica. Madrid: Instituto de Acústica, Consejo Superior de Investigaciones Científicas.

(28) Ayuntamiento de Zaragoza. (2009). Mapas de niveles sonoros LDia. https://www.zaragoza.es/ciudad/medioambiente/ atmosfera/maparuido/planos10.htm.

(29) Maciá, M. E., Rolando, A. (2013). Variación del módulo de Young de un elemento de fábrica de ladrillo sometido a altas temperaturas. Materiales de Construcción, 63(309): 105-116, doi: http://dx.doi.org/10.3989/mc.2012.02311.

(30) Rosell, J. R., Cantalapiedra, I. R. (2011). Método simple para determinar el módulo de Young dinámico a partir de una excitación por impacto aplicado a morteros de cal y cemento. Materiales de Construcción, 61(301): 39-48, doi: http://dx.doi.org/10.3989/mc.2010.53509.

(31) Flores, V., Sánchez-Tizapa, S., Arroyo, R., Barragán, R. (2013). Propiedades mecánicas de la mampostería de tabique rojo recocido utilizada en Chilpancingo, Gro (México). Informes de la Construcción, 65(531): 387-395, doi: http://dx.doi.org/10.3989/ic.12.084. 